Prepared in cooperation with the U.S. Fish and Wildlife Service

Optimization of Salt Marsh Management at the Eastern Shore of Virginia and Fisherman Island National Wildlife Refuges, Virginia, Through Use of Structured Decision Making

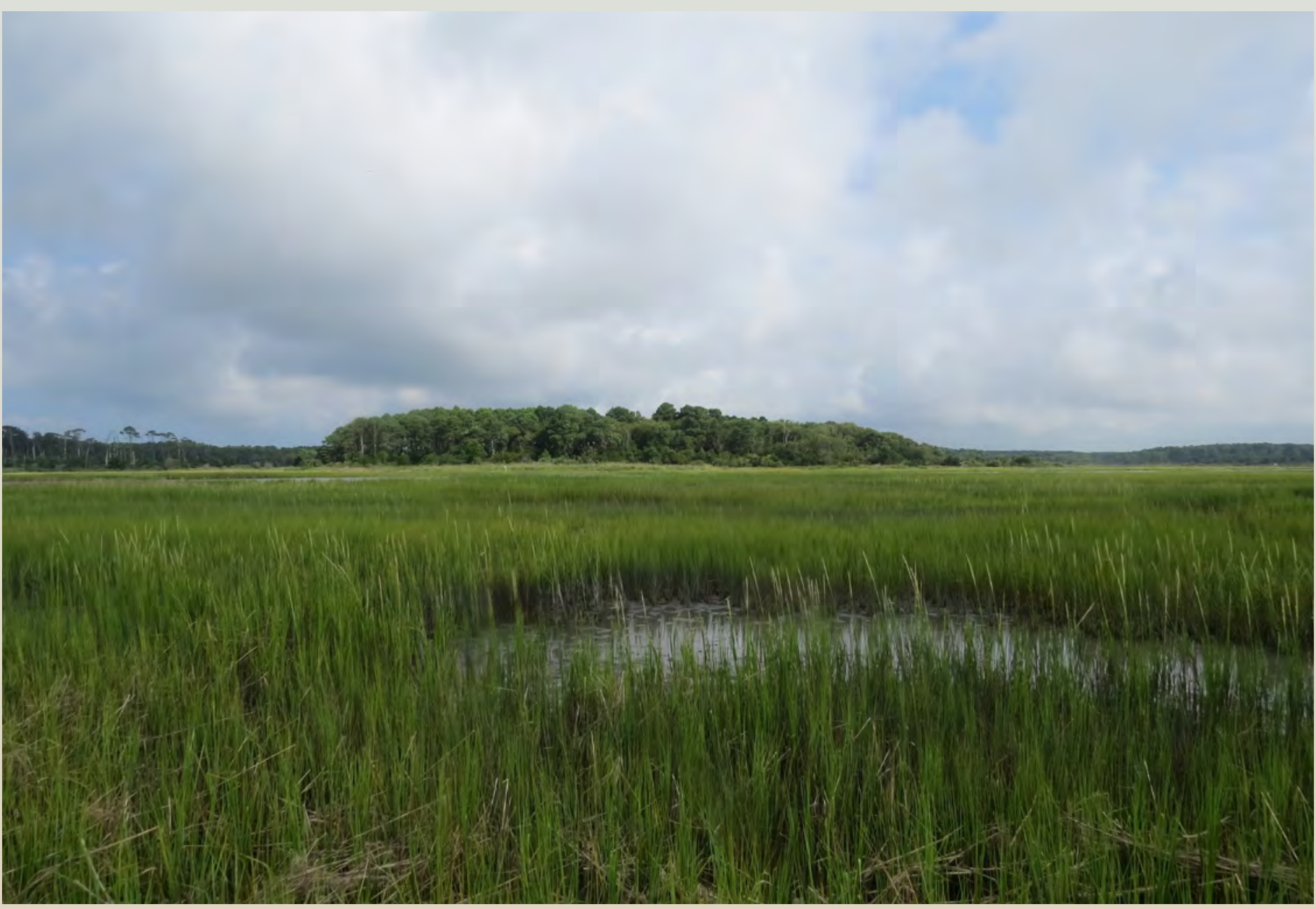

Open-File Report 2021-1117 
Photograph of ESV Marsh in the Eastern Shore of Virginia National Wildlife Refuge, Cape Charles, Virginia; photograph by the U.S. Fish and Wildlife Service. 


\section{Optimization of Salt Marsh Management at the Eastern Shore of Virginia and Fisherman Island National Wildlife Refuges, Virginia, Through Use of Structured Decision Making}

By Hilary A. Neckles, James E. Lyons, Jessica L. Nagel, Susan C. Adamowicz, Toni Mikula, Pamela Denmon, and Robert Leffel

Prepared in cooperation with the U.S. Fish and Wildlife Service

Open-File Report 2021-1117 


\section{U.S. Geological Survey, Reston, Virginia: 2021}

For more information on the USGS - the Federal source for science about the Earth, its natural and living resources, natural hazards, and the environment—visit https://www.usgs.gov or call 1-888-ASK-USGS.

For an overview of USGS information products, including maps, imagery, and publications, visit https://store.usgs.gov/.

Any use of trade, firm, or product names is for descriptive purposes only and does not imply endorsement by the U.S Government.

The findings and conclusions in this report are those of the authors and do not necessarily represent the views of the U.S. Fish and Wildlife Service.

Although this information product, for the most part, is in the public domain, it also may contain copyrighted materials as noted in the text. Permission to reproduce copyrighted items must be secured from the copyright owner.

Suggested citation:

Neckles, H.A., Lyons, J.E., Nagel, J.L., Adamowicz, S.C., Mikula, T., Denmon, P., and Leffel, R., 2021, Optimization of salt marsh management at the Eastern Shore of Virginia and Fisherman Island National Wildlife Refuges, Virginia, through use of structured decision making: U.S. Geological Survey Open-File Report 2021-1117, 32 p., https://doi.org/ 10.3133/ofr20211117.

Associated data for this publication:

U.S. Fish and Wildlife Service, 2016, Salt marsh integrity and Hurricane Sandy vegetation, bird and nekton data: U.S. Fish and Wildlife Service database, https://ecos.fws.gov/ServCat/Reference/Profile/121918.

ISSN 2331-1258 (online) 


\section{Acknowledgments}

The U.S. Fish and Wildlife Service (FWS) provided exceptional hospitality at the Eastern Shore of Virginia National Wildlife Refuge, Cape Charles, Virginia, for the 2018 structured decisionmaking workshop, which included participants from two additional national wildlife refuge administrative units. Nathan Bush of the FWS generated the mapping data used in this report, and Jackie Olson of the U.S. Geological Survey expertly prepared the maps. Technical reviews by Rachel Katz of the FWS and Bill Thompson of the National Park Service greatly improved the quality of this report. 



\section{Contents}

Acknowledgments ……...................................................................................................................

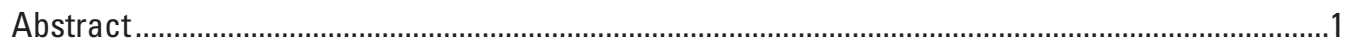

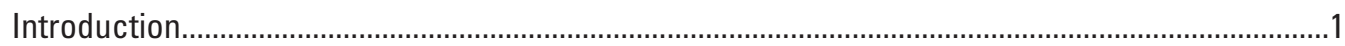

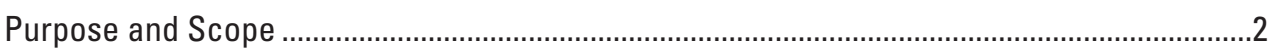

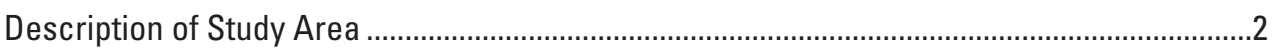

Regional Structured Decision-Making Framework ……………...................................................

Application to the Eastern Shore of Virginia and Fisherman Island

National Wildlife Refuges ...........................................................................................

Results of Constrained Optimization......................................................................................15

Considerations for Optimizing Salt Marsh Management............................................................17

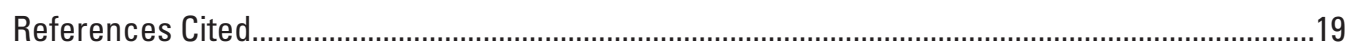

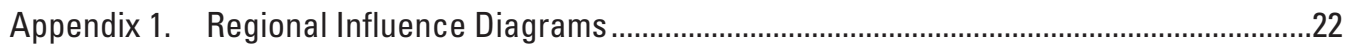

Appendix 2. Utility Functions for the Eastern Shore of Virginia and Fisherman Island National Wildlife Refuges

\section{Figures}

1. Map showing national wildlife refuges and national wildlife refuge complexes of the U.S. Fish and Wildlife Service where salt marsh integrity was assessed from 2012 to 2016 using the regional monitoring protocol.

2. Map showing salt marsh management units at the Eastern Shore of Virginia and Fisherman Island National Wildlife Refuges in Virginia

3. Graph showing predicted total management benefit of various portfolios, expressed as weighted utilities, relative to total cost at the Eastern Shore of Virginia and Fisherman Island National Wildlife Refuges in Virginia

4. Bar chart of predicted management benefit of portfolio 5 in comparison to the management benefit from the baseline "no-action" portfolio at the Eastern Shore of Virginia and Fisherman Island National Wildlife Refuges in Virginia

\section{Tables}

1. Objectives hierarchy for salt marsh management decision problems

2. Participants in workshop convened at the Eastern Shore of Virginia National Wildlife Refuge to apply a regional framework for optimizing salt marsh management decisions to three national wildlife refuge administrative units in February 2018

3. Possible management actions for achieving objectives within marsh management units at the Eastern Shore of Virginia and Fisherman Island National Wildlife Refuges, Virginia, estimated costs over 5 years, and predicted outcomes expressed relative to performance metrics

4. Normalized predicted outcomes and estimated total management benefits of possible management actions within six marsh management units at the Eastern Shore of Virginia and Fisherman Island NationalWildlife Refuges, Virginia

5. Actions included in various management portfolios to maximize the total management benefits subject to increasing cost constraints at the Eastern Shore of Virginia and Fisherman Island National Wildlife Refuges, Virginia 


\section{Conversion Factors}

International System of Units to U.S. customary units

\begin{tabular}{|c|c|c|}
\hline Multiply & By & To obtain \\
\hline meter $(\mathrm{m})$ & 3.281 & foot $(\mathrm{ft})$ \\
\hline kilometer (km) & 0.6214 & mile (mi) \\
\hline square meter $\left(\mathrm{m}^{2}\right)$ & 0.0002471 & acre \\
\hline hectare (ha) & 2.471 & acre \\
\hline
\end{tabular}

\section{Datums}

Vertical coordinate information is referenced to the North American Vertical Datum of 1988 (NAVD 88).

Horizontal coordinate information is referenced to the North American Datum of 1983 (NAD 83).

Elevation, as used in this report, refers to distance above the vertical datum.

\section{Abbreviations}

FWS U.S. Fish and Wildlife Service

NWR national wildlife refuge

USGS U.S. Geological Survey 


\title{
Optimization of Salt Marsh Management at the Eastern Shore of Virginia and Fisherman Island National Wildlife Refuges, Virginia, Through Use of Structured Decision Making
}

\author{
By Hilary A. Neckles, ${ }^{1}$ James E. Lyons, ${ }^{1}$ Jessica L. Nagel, ${ }^{1}$ Susan C. Adamowicz, ${ }^{2}$ Toni Mikula, ${ }^{2}$ \\ Pamela Denmon, ${ }^{2}$ and Robert Leffe ${ }^{2}$
}

\section{Abstract}

Structured decision making is a systematic, transparent process for improving the quality of complex decisions by identifying measurable management objectives and feasible management actions; predicting the potential consequences of management actions relative to the stated objectives; and selecting a course of action that maximizes the total benefit achieved and balances tradeoffs among objectives. The U.S. Geological Survey, in cooperation with the U.S. Fish and Wildlife Service, applied an existing, regional framework for structured decision making to develop a prototype tool for optimizing tidal marsh management decisions at the Eastern Shore of Virginia and Fisherman Island National Wildlife Refuges in Virginia. Refuge biologists, refuge managers, and research scientists identified multiple potential management actions to improve the ecological integrity of six marsh management units within the refuges, totaling about 575 hectares, and estimated the outcomes of each action in terms of performance metrics associated with each management objective. Value functions previously developed at the regional level were used to transform metric scores to a common utility scale, and utilities were summed to produce a single score representing the total management benefit that could be accrued from each potential management action. Constrained optimization was used to identify the set of management actions, one per marsh management unit, that could maximize total management benefits at different cost constraints at the refuge scale. Results indicated that, for the objectives and actions considered here, total management benefits may increase consistently up to approximately $\$ 143,000$, but that further expenditures may yield diminishing return on investment. Potential management actions in optimal portfolios at total costs less than $\$ 143,000$ included digging runnels by hand to improve drainage from the marsh surface, breaching a road to restore natural hydrology, trapping predators to enhance nest success of tidal marsh birds, and reducing the abundance

${ }^{1}$ U.S. Geological Survey.

${ }^{2}$ U.S. Fish and Wildlife Service. of Odocoileus virginianus (white-tailed deer) to minimize their effects on marsh vegetation. The potential management benefits were derived from expected increases in number of tidal marsh obligate breeding birds, species richness of nekton, and density of spiders (as an indicator of trophic health); and an expected decrease in duration of surface flooding. The prototype presented here does not resolve management decisions; rather, it provides a framework for decision making at the Eastern Shore of Virginia and Fisherman Island National Wildlife Refuges that can be updated as new data and information become available. Insights from this process may also be useful to inform future habitat management planning at the refuges.

\section{Introduction}

The National Wildlife Refuge System protects extensive salt marsh acreage in the northeastern United States. Much of this habitat has been degraded by a succession of human activities since the time of European settlement (Gedan and others, 2009), and accelerated rates of sea-level rise exacerbate these effects (Gedan and others, 2011; Kirwan and Megonigal, 2013). Therefore, strategies to restore and enhance the ecological integrity of national wildlife refuge (NWR) salt marshes are regularly considered. Management may include such activities as reestablishing natural hydrology, augmenting or excavating sediments to restore marsh elevation, controlling invasive species, planting native vegetation, minimizing shoreline erosion, and remediating contaminant problems. Uncertainty stemming from incomplete knowledge of system status and imperfect understanding of ecosystem dynamics commonly hinders management predictions and selection of the most effective management options. Consequently, tools for identifying appropriate assessment variables and evaluating tradeoffs among management objectives are valuable to inform marsh management decisions.

Structured decision making is a systematic approach to improving the quality of complex decisions that integrates assessment metrics into the decision process 
(Gregory and Keeney, 2002). This approach involves identifying measurable management objectives and potential management actions, predicting management outcomes, and evaluating tradeoffs to choose a preferred alternative. From 2008 to 2012, the U.S. Geological Survey (USGS) and U.S. Fish and Wildlife Service (FWS) used structured decision making to develop a framework for optimizing management decisions for NWR salt marshes in the FWS Northeast Region (that is, salt marshes in the coastal region from Maine through Virginia). The structured decision-making steps were applied through successive "rapid prototyping" workshops, an iterative process in which relatively short periods of time are invested to continually improve the decision structure (Blomquist and others, 2010; Garrard and others, 2017). The decision framework includes regional management objectives addressing critical components of salt marsh ecosystems, and associated performance metrics for determining whether objectives are achieved (Neckles and others, 2015). The regional objectives structure served as the foundation for a consistent protocol for monitoring salt marsh integrity at these northeastern coastal refuges, in which the monitoring variables are linked explicitly to management goals (Neckles and others, 2013). From 2012 to 2016, this protocol was used to conduct a baseline assessment of salt marsh integrity at all 17 refuges or refuge complexes in the FWS Northeast Region with salt marsh habitat (fig. 1).

The Eastern Shore of Virginia National Wildlife Refuge protects 180 hectares (ha) of salt marsh at the southern end of the Delmarva Peninsula, in Northampton County, Virginia, between the Atlantic Ocean and Chesapeake Bay (fig. 2). The Fisherman Island National Wildlife Refuge protects an additional 393 ha of salt marsh on a barrier island separated from the Delmarva Peninsula by Fishermans Inlet (fig. 2). The two refuges are administered jointly and are considered together for descriptive and management purposes. The salt marsh on the refuges provides critical breeding, migratory, or wintering habitat for bird species of highest conservation priority, including Anas rubripes (American black duck), Branta bernicla (Atlantic brant), and Ammodramus caudacutus (saltmarsh sparrow) in the New England and mid-Atlantic coast bird conservation region of the U.S. North American Bird Conservation Initiative (Steinkamp, 2008; Denmon, 2018; U.S. North American Bird Conservation Initiative, 2020). The salt marsh also provides important foraging habitat for wading birds, such as Ardea alba (great egret) and Egretta thula (snowy egret), during the breeding season. The primary threats to this habitat are marsh submergence associated with rising sea level, spread of the invasive reed Phragmites australis (hereafter referred to as Phragmites), and overabundance of Odocoileus virginianus (white-tailed deer; Denmon, 2018). Salt-marsh management goals set by the FWS for the refuges include maintaining the long-term productivity, integrity, and function of this habitat for marsh-dependent birds (FWS, 2004; Denmon, 2018). In this study, the regional structured decision-making framework was used to help prioritize salt marsh management options for the refuges.

\section{Purpose and Scope}

This report describes the application of the regional structured decision-making framework (Neckles and others, 2015) to the Eastern Shore of Virginia and Fisherman Island National Wildlife Refuges. The regional framework was parameterized to local conditions through rapid prototyping, producing a decision model for the refuges that can be updated as new information becomes available. Included are a suite of potential management actions to achieve objectives in six marsh management units of the refuges (four units at the Eastern Shore of Virginia National Wildlife Refuge and two units at the Fisherman Island National Wildlife Refuge; fig. 2), approximate costs for implementing each potential action, predictions for the outcome of each management action relative to individual management objectives, and results of constrained optimization to maximize management benefits subject to cost constraints. This decision structure can be used to understand how specific actions may contribute to achieving management objectives and identify an optimum combination of actions, or "management portfolio," to maximize management benefits at the refuge scale for a range of potential budgets. The prototype presented here provides a framework for continually improving the quality of complex management decisions at the Eastern Shore of Virginia and Fisherman Island National Wildlife Refuges.

\section{Description of Study Area}

The Eastern Shore of Virginia and Fisherman Island National Wildlife Refuges are located at the southern tip of the Delmarva Peninsula (fig. 1), an area that concentrates millions of birds annually during southward migration. The refuges' habitats, including maritime forest, shrub thickets, grasslands, beaches, and salt marsh, provide some of the country's most valuable stopovers for migratory birds (FWS, 2004; Denmon, 2018). The salt marsh at the Eastern Shore of Virginia National Wildlife Refuge is divided into four marsh management units ranging in size from 22 ha to $76 \mathrm{ha}$, and the salt marsh at the Fisherman Island National Wildlife Refuge is divided into two marsh management units at 54 ha and 339 ha in size (fig. 2). At both refuges, the primary salt marsh habitat is low marsh dominated by Spartina alterniflora (smooth cordgrass), which is flooded regularly at high tide. The salt marshes throughout the refuges are relatively unaltered by historic human activities such as ditching. The majority of the area within 150-meter and 1-kilometer buffers around the marsh management unit boundaries is categorized under natural land uses (land classified by the 2011 National Land Cover Database as categories other than agricultural or developed; Multi-Resolution Land Characteristics Consortium, 2020). During summertime sampling in 2014 and 2015, average surface-water salinities ranged from about 26 to 35 parts per thousand (polyhaline to euhaline as defined by Cowardin and others, 1979) within the marsh management units (FWS, 2016). 


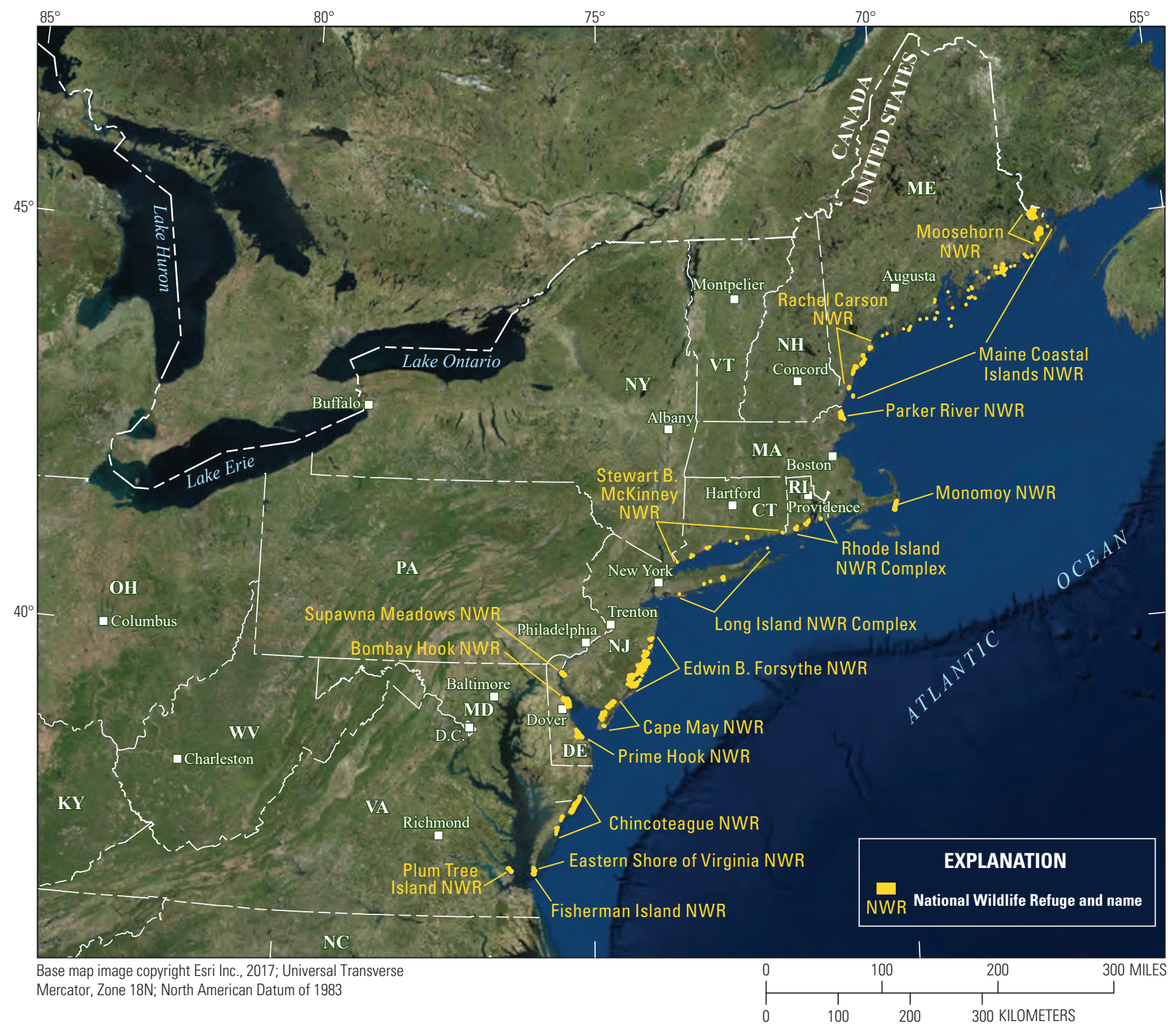

Figure 1. Map showing national wildlife refuges and national wildlife refuge complexes of the U.S. Fish and Wildlife Service where salt marsh integrity was assessed from 2012 to 2016 using the regional monitoring protocol. 


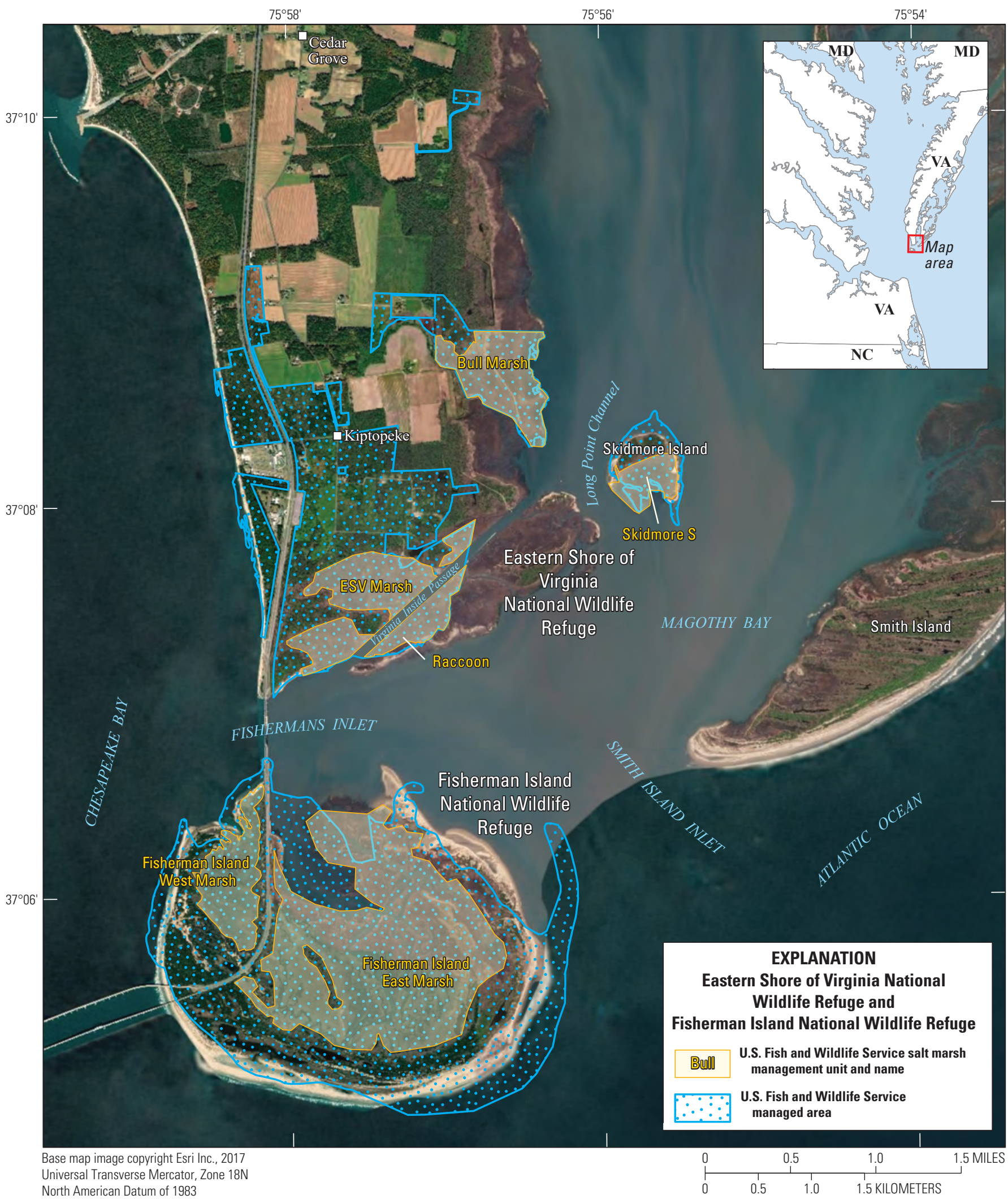

Figure 2. Map showing salt marsh management units at the Eastern Shore of Virginia and Fisherman Island National Wildlife Refuges in Virginia. U.S. Fish and Wildlife Service managed areas shown for reference. 


\section{Regional Structured Decision-Making Framework}

A regional framework for assessing and managing salt marsh integrity at northeastern NWRs was developed through collaborative efforts of FWS regional and refuge managers and biologists, salt marsh research scientists, and structured decision-making experts. This process followed the discrete steps outlined by Hammond and others (1999) and Gregory and Keeney (2002):

1. Clarify the temporal and spatial scope of the management decision.

2. Define objectives and performance measures to evaluate whether objectives are achieved.

3. Develop alternative management actions for achieving objectives.

4. Estimate the consequences or likely outcomes of management actions in terms of the performance measures.

5. Evaluate the tradeoffs inherent in potential alternatives and select the optimum alternatives to maximize management benefits.

This sequence of steps was applied through successive workshops to refine the decision structure and incorporate newly available information. Initial development of the structured decision-making framework occurred during a weeklong workshop in 2008 to define the decision problem, specify management objectives, and explore potential strategies available to restore and enhance salt marsh integrity. During 2008 and 2009, workshop results were used to guide field tests of salt marsh monitoring variables (Neckles and others, 2013). Subsequently, in 2012, data and insights gained from these field tests were used in a two-part workshop to refine management objectives and develop the means for evaluating management outcomes (Neckles and others, 2015).

From the outset, FWS goals included development of an approach for consistent assessment of salt marsh integrity across all northeastern NWRs (fig. 1). Within this regional context, staff at a given refuge must periodically determine the best approaches for managing salt marshes to maximize habitat value while considering financial and other constraints.
The salt marsh decision problem was thus defined as applying to individual NWRs over a 5-year planning horizon. The objectives for complex decisions can be organized into a hierarchy to help clarify what is most important to decision makers (Gregory and others, 2012). The hierarchy of objectives for salt marsh management decisions (table 1) was based explicitly on the conservation mission of the National Wildlife Refuge System, which is upheld through FWS management to "ensure that the biological integrity, diversity, and environmental health of the System are maintained for the benefit of present and future generations of Americans," as mandated in the National Wildlife Refuge System Improvement Act of 1997 (16 U.S.C. §668dd note). Two fundamental objectives, or the overall goals for salt marsh management decisions, were drawn from this policy to maximize (1) biological integrity and diversity, and (2) environmental health, of salt marsh ecosystems. Participants in the prototyping workshops deconstructed these overall goals into lower-level objectives relating to salt marsh structure and function and identified performance metrics to evaluate whether objectives are achieved (table 1). In addition, performance metrics were weighted to reflect the relative importance of each objective (Neckles and others, 2015).

The hierarchy of objectives for salt marsh management (table 1) provides the foundation for identifying possible management actions at individual NWRs and predicting management outcomes. Workshop participants developed preliminary influence diagrams (app. 1), or conceptual models relating management actions to responses by each performance metric (Conroy and Peterson, 2013), to guide this process. To allow metric responses to be aggregated into a single, overall performance score, participants also defined value functions relating salt marsh integrity metric scores to perceived management benefit on a common, unitless "utility" scale (Keeney and Raiffa, 1993). Stakeholder elicitation was used to determine the form of each value function relating the original metric scale to the utility scale, ranging from 0 , representing the lowest management benefit, to 1 , representing the highest benefit (app. 2). Neckles and others (2015) provided details regarding development of the structured decision-making framework and a case-study application to Prime Hook National Wildlife Refuge in Delaware. 
Table 1. Objectives hierarchy for salt marsh management decision problems.

[Two fundamental objectives (overall goals of the decision problem) draw directly from U.S. Fish and Wildlife Service (FWS) National Wildlife Refuge System policy to maintain, restore, and enhance biological integrity, diversity, and environmental health within the refuges. These are broken down into lower level objectives focused on specific aspects of marsh structure and function. Values in parentheses are weights assigned to objectives, reflecting their relative importance. Weights on any branch of the hierarchy (that is, objectives that are at the same level of the hierarchy under a fundamental objective) sum to one. The weight for each metric is the product of the weights from each level of the hierarchy leading to that metric. See also Neckles and others (2015). NA, not applicable]

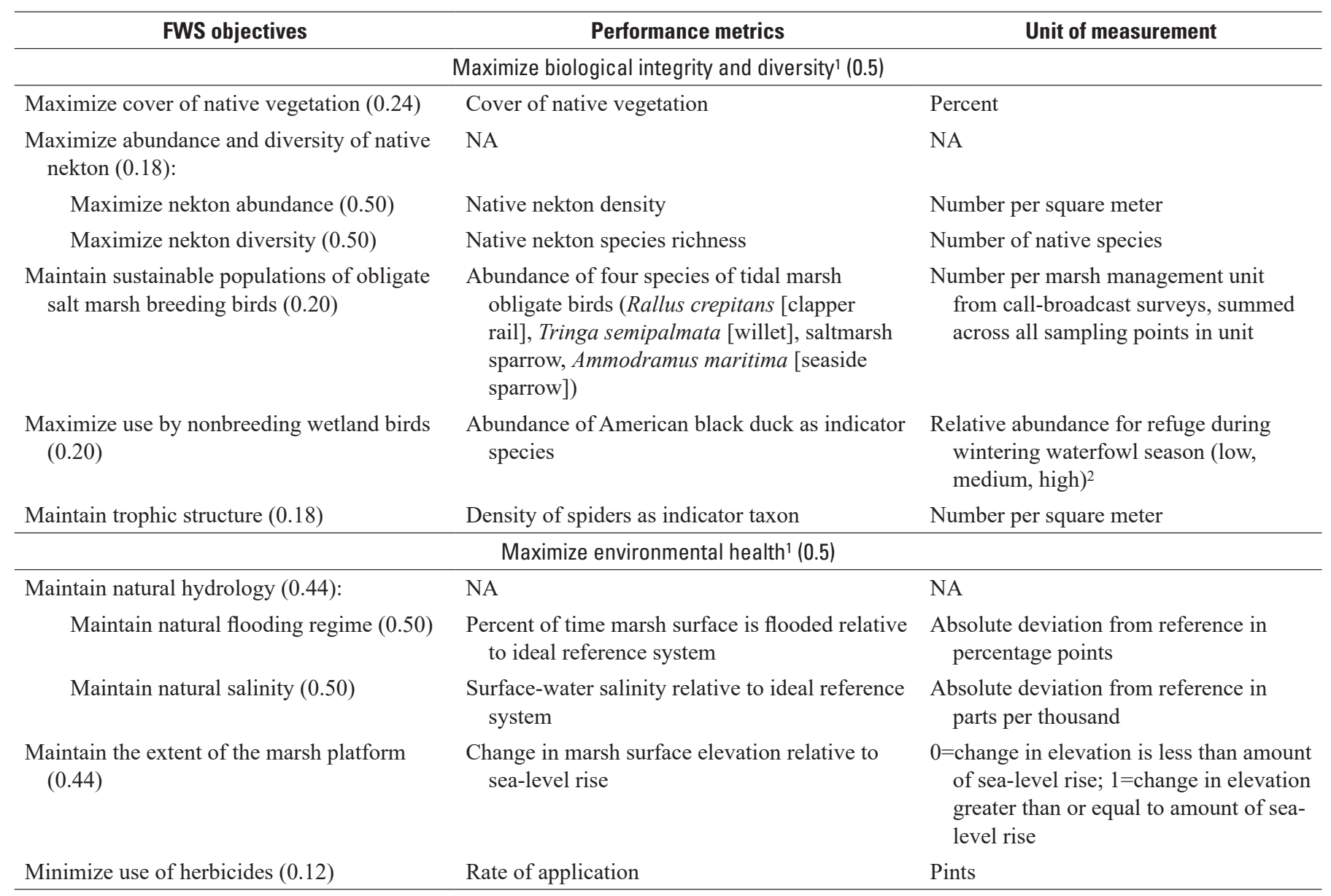

${ }^{1}$ Fundamental objectives of salt marsh management decisions.

${ }^{2}$ Relative abundance based on local knowledge. 


\section{Application to the Eastern Shore of Virginia and Fisherman Island National Wildlife Refuges}

In February 2018, FWS regional biologists, biologists and managers from four northeastern NWR administrative units, and USGS research scientists (table 2) participated in a 1.5-day rapid-prototyping workshop to apply the regional structured decision-making framework to the Eastern Shore of Virginia, Fisherman Island, and Plum Tree Island National Wildlife Refuges and the Long Island National Wildlife Refuge Complex. Participants worked within refuge-specific small groups to focus on management issues at individual refuges. Plenary discussions of common patterns of salt marsh degradation, potential management strategies, and mechanisms of ecosystem response offered additional insights to enhance refuge-specific discussions.

Participants identified a range of possible management actions for achieving objectives within each marsh management unit at the Eastern Shore of Virginia and Fisherman Island National Wildlife Refuges and estimated the total cost of implementation over a 5-year period; the specific years of implementation were not identified in this prototype. Potential actions to enhance salt marsh integrity ranged from targeted efforts that restore hydrologic connections, increase surface-water drainage, or control predators, deer, or spread of Phragmites, to large-scale projects that alter marsh elevation (table 3). Participants predicted the outcomes of each

Table 2. Participants in workshop convened at the Eastern Shore of Virginia National Wildlife Refuge to apply a regional framework for optimizing salt marsh management decisions to three national wildlife refuge administrative units in February 2018.

[FWS, U.S. Fish and Wildlife Service; NWR, National Wildlife Refuge; USGS, U.S. Geological Survey]

\begin{tabular}{ll}
\hline \multicolumn{1}{c}{ Affiliation } & \multicolumn{1}{c}{ Participant } \\
\hline \multicolumn{1}{c}{ FWS NWR specialists } \\
$\begin{array}{l}\text { Eastern Shore of Virginia and Fisherman } \\
\text { Island NWRs }\end{array}$ & Pam Denmon \\
$\begin{array}{l}\text { Eastern Shore of Virginia and Fisherman } \\
\text { Island NWRs }\end{array}$ & Robert Leffel \\
Long Island NWR Complex & Monica Williams \\
Plum Tree Island NWR & William Crouch \\
Plum Tree Island NWR & Lauren Cruz \\
\hline \multicolumn{2}{c}{ FWS regional expert } \\
\hline Rachel Carson NWR & Susan Adamowicz \\
\hline \multicolumn{2}{c}{ Research scientists } \\
\hline USGS Eastern Ecological Science Center & James Lyons \\
USGS Eastern Ecological Science Center & Hilary Neckles \\
\hline
\end{tabular}

management action 5 years after initial implementation in terms of salt marsh integrity performance metrics. For most metrics, baseline conditions within each unit measured during the 2012-16 salt marsh integrity assessment (FWS, 2016) were used to predict the outcomes of a "no-action" alternative. Baseline conditions were estimated by using expert judgement for three metrics that lacked assessment data (abundance of American black ducks, density of spiders, change in marsh surface elevation relative to sea-level rise). Regional influence diagrams relating management strategies to outcomes aided in predicting consequences of management actions (app. 1). Although the influence diagrams incorporated the potential effects of stochastic processes, including weather, sea-level rise, herbivory, contaminant inputs, and disease, on management outcomes, no attempt was made to quantify these sources of uncertainty during rapid prototyping. Management predictions also inherently included considerable uncertainty surrounding the complex interactions among controlling factors and salt marsh ecosystem components.

Following the workshop, the potential management benefit of each salt marsh integrity performance metric was calculated by converting salt marsh integrity metric scores (table 3, workshop output) to weighted utilities (table 4) using regional value functions (app. 2). Weighted utilities were summed across all salt marsh integrity metrics for each action; this overall utility therefore represented the total management benefit, across all objectives, expected to accrue from a given management action (table 4). Constrained optimization (Conroy and Peterson, 2013) was used to find the management portfolio (the combination of actions, one action per marsh management unit) that maximizes the total management benefit across all units under varying cost scenarios for the combined refuges. Constrained optimization using integer linear programming was implemented in the Solver tool in Microsoft Excel (Kirkwood, 1997). Budget constraints were increased in $\$ 25,000$ increments up to $\$ 50,000$; in $\$ 50,000$ increments up to $\$ 300,000$; in $\$ 100,000$ increments up to $\$ 1$ million; in $\$ 500,000$ increments up to $\$ 3$ million; and in $\$ 1$ million increments thereafter. The upper limit to potential costs was not determined in advance; rather, it reflected the total estimated costs of the proposed management actions. A cost-benefit plot of the portfolios identified through the optimization analysis was used to identify the efficient frontier for resource allocation (Keeney and Raiffa, 1993), which is the set of portfolios that are not dominated by other portfolios at similar costs (or the set of portfolios with maximum total benefit for a similar cost). The cost-benefit plot also revealed the cost above which further expenditures would yield diminishing returns on investment. To exemplify use of the decision-making framework to understand how a given portfolio could affect specific management objectives, the refuge-scale management benefits for individual performance metrics were compared between one optimal portfolio and those predicted with no management action taken. 
Table 3. Possible management actions for achieving objectives within marsh management units at the Eastern Shore of Virginia and Fisherman Island National Wildlife Refuges, Virginia, estimated costs over 5 years, and predicted outcomes expressed relative to performance metrics.

[Potential management actions, costs, and predicted outcomes developed by workshop participants using expert judgement. Predicted consequences of management actions aided by influence diagrams (app. 1). $\%$, percent; ppt, parts per thousand]

\begin{tabular}{|c|c|c|c|c|c|c|c|c|c|c|c|}
\hline \multirow[b]{3}{*}{ Management action } & \multirow[b]{3}{*}{$\begin{array}{l}\text { Estimated } \\
\text { cost over } \\
5 \text { years } \\
\text { (dollars) }\end{array}$} & \multicolumn{10}{|c|}{ Performance metrics } \\
\hline & & \multirow[b]{2}{*}{$\begin{array}{l}\text { Native } \\
\text { vegetation } \\
\text { (\% cover) }\end{array}$} & \multicolumn{2}{|c|}{ Nekton } & \multirow[b]{2}{*}{$\begin{array}{c}\text { Tidal } \\
\text { marsh obli- } \\
\text { gate birds } \\
\text { (summed } \\
\text { number per } \\
\text { point) }\end{array}$} & \multirow[b]{2}{*}{$\begin{array}{l}\text { American } \\
\text { black } \\
\text { ducks } \\
\text { use }^{1}\end{array}$} & \multirow[b]{2}{*}{$\begin{array}{c}\text { Spider } \\
\text { density } \\
\text { (number } \\
\text { per } \\
\text { square } \\
\text { meter) }\end{array}$} & \multicolumn{2}{|c|}{ Hydrology } & \multirow{2}{*}{$\begin{array}{c}\text { Marsh } \\
\text { surface } \\
\text { elevation } \\
\text { change } \\
\text { relative to } \\
\text { sea-level } \\
\text { rise }^{3}\end{array}$} & \multirow[b]{2}{*}{$\begin{array}{l}\text { Herbicide } \\
\text { appli- } \\
\text { cation } \\
\text { (pints/ } \\
\text { year) }\end{array}$} \\
\hline & & & $\begin{array}{c}\text { Density } \\
\text { (number } \\
\text { of animals } \\
\text { per square } \\
\text { meter) }\end{array}$ & $\begin{array}{l}\text { Species } \\
\text { richness } \\
\text { (number) }\end{array}$ & & & & $\begin{array}{l}\text { Duration } \\
\text { of surface } \\
\text { flooding }{ }^{2} \\
(\%)\end{array}$ & $\begin{array}{l}\text { Surface- } \\
\text { water } \\
\text { salinity } \\
\text { (ppt) }\end{array}$ & & \\
\hline \multicolumn{12}{|c|}{ Bull Marsh } \\
\hline A. No action & 0 & 98 & 76 & 7 & 3.5 & Medium & 30 & 10.6 & 6 & 1 & 0 \\
\hline $\begin{array}{l}\text { B. Remove road and control any resulting } \\
\text { invasive species }\end{array}$ & 250,000 & 100 & 91 & 10 & 5.5 & Medium & 30 & 0 & 6 & 1 & 6 \\
\hline $\begin{array}{l}\text { C. Breach road in four places and control any } \\
\text { resulting invasive species }\end{array}$ & 20,000 & 100 & 84 & 9 & 4.5 & Medium & 30 & 5 & 6 & 1 & 3 \\
\hline D. Lower road elevation & 50,000 & 100 & 80 & 8 & 4.5 & Medium & 30 & 7 & 6 & 1 & 0 \\
\hline $\begin{array}{l}\text { E. Remove Phragmites in western edge of unit } \\
\text { and field }\end{array}$ & 25,000 & 100 & 76 & 7 & 3.6 & Medium & 30 & 10.6 & 6 & 1 & 60 \\
\hline $\begin{array}{l}\text { F. Facilitate marsh migration by leveling } \\
\text { berms and ditches west of unit and control- } \\
\text { ling invasive plants }\end{array}$ & 15,000 & 100 & 76 & 7 & 3.5 & Medium & 30 & 9 & 6 & 1 & 12 \\
\hline G. Create more pools and runnels & 60,000 & 100 & 100 & 8 & 3.5 & Medium & 15 & 10.6 & 6 & 1 & 0 \\
\hline $\begin{array}{l}\text { H. Trap mammalian predators, including } \\
\text { Procyon lotor (raccoon), to increase nesting } \\
\text { success of tidal marsh birds }\end{array}$ & 50,000 & 100 & 76 & 7 & 4 & Medium & 30 & 10.6 & 6 & 1 & 0 \\
\hline I. Reduce disturbance through refuge permits & 7,800 & 100 & 76 & 7 & 3.7 & Medium & 30 & 10.6 & 6 & 1 & 0 \\
\hline J. Acquire land and control Phragmites & 17,400 & 98 & 108 & 9 & 3.6 & Medium & 30 & 9 & 6 & 1 & 30 \\
\hline $\begin{array}{l}\text { K. Install three culverts in road and control } \\
\text { invasive plants }\end{array}$ & 20,000 & 100 & 82 & 9 & 4.5 & Medium & 30 & 4 & 6 & 1 & 3 \\
\hline L. B $+\mathrm{G}$ & 310,000 & 100 & 130 & 11 & 5.7 & Medium & 15 & 10.6 & 6 & 1 & 0 \\
\hline \multicolumn{12}{|c|}{ Skidmore S } \\
\hline A. No action & 0 & 99 & 12 & 6 & 0.69 & Medium & 15 & 22.8 & 2 & 1 & 0 \\
\hline $\begin{array}{l}\text { B. Create islands for marsh bird nesting using } \\
\text { dredge spoils }\end{array}$ & 30,000 & 98 & 12 & 6 & 1 & Medium & 15 & 18 & 2 & 1 & 0 \\
\hline $\begin{array}{l}\text { C. Apply broad-scale sediment to marsh plat- } \\
\text { form to lower flood duration }\end{array}$ & 90,000 & 99 & 11 & 6 & 1 & Medium & 30 & 0 & 2 & 1 & 0 \\
\hline
\end{tabular}




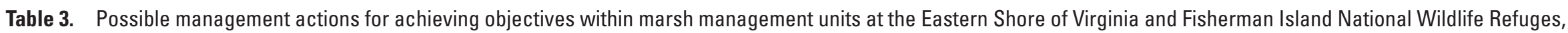
Virginia, estimated costs over 5 years, and predicted outcomes expressed relative to performance metrics.-Continued

[Potential management actions, costs, and predicted outcomes developed by workshop participants using expert judgement. Predicted consequences of management actions aided by influence diagrams (app. 1). $\%$, percent; ppt, parts per thousand]

\begin{tabular}{|c|c|c|c|c|c|c|c|c|c|c|c|}
\hline \multirow[b]{3}{*}{ Management action } & \multirow[b]{3}{*}{$\begin{array}{l}\text { Estimated } \\
\text { cost over } \\
5 \text { years } \\
\text { (dollars) }\end{array}$} & \multicolumn{10}{|c|}{ Performance metrics } \\
\hline & & \multirow[b]{2}{*}{$\begin{array}{l}\text { Native } \\
\text { vegetation } \\
\text { (\% cover) }\end{array}$} & \multicolumn{2}{|c|}{ Nekton } & \multirow[b]{2}{*}{$\begin{array}{c}\text { Tidal } \\
\text { marsh obli- } \\
\text { gate birds } \\
\text { (summed } \\
\text { number per } \\
\text { point) }\end{array}$} & \multirow[b]{2}{*}{$\begin{array}{l}\text { American } \\
\text { black } \\
\text { ducks }^{1} \\
\text { use }^{1}\end{array}$} & \multirow[b]{2}{*}{$\begin{array}{c}\text { Spider } \\
\text { density } \\
\text { (number } \\
\text { per } \\
\text { square } \\
\text { meter) }\end{array}$} & \multicolumn{2}{|c|}{ Hydrology } & \multirow{2}{*}{$\begin{array}{l}\text { Marsh } \\
\text { surface } \\
\text { elevation } \\
\text { change } \\
\text { relative to } \\
\text { sea-level } \\
\text { rise }^{3}\end{array}$} & \multirow[b]{2}{*}{$\begin{array}{c}\text { Herbicide } \\
\text { appli- } \\
\text { cation } \\
\text { (pints/ } \\
\text { year) }\end{array}$} \\
\hline & & & $\begin{array}{c}\text { Density } \\
\text { (number } \\
\text { of animals } \\
\text { per square } \\
\text { meter) }\end{array}$ & $\begin{array}{l}\text { Species } \\
\text { richness } \\
\text { (number) }\end{array}$ & & & & $\begin{array}{c}\text { Duration } \\
\text { of surface } \\
\text { flooding } \\
(\%)\end{array}$ & $\begin{array}{l}\text { Surface- } \\
\text { water } \\
\text { salinity }{ }^{2} \\
\text { (ppt) }\end{array}$ & & \\
\hline \multicolumn{12}{|c|}{ Skidmore S-Continued } \\
\hline $\begin{array}{l}\text { D. Remove Phragmites on uplands and marsh } \\
\text { edge }\end{array}$ & 7,500 & 100 & 12 & 6 & 0.7 & Medium & 15 & 22.8 & 2 & 1 & 18 \\
\hline $\begin{array}{l}\text { E. Trap mammalian predators, including rac- } \\
\text { coons, to increase nesting success of tidal } \\
\text { marsh birds }\end{array}$ & 50,000 & 100 & 12 & 6 & 1.2 & Medium & 15 & 22.8 & 2 & 1 & 0 \\
\hline F. B+E & 80,000 & 99 & 12 & 6 & 2 & Medium & 15 & 18 & 2 & 1 & 0 \\
\hline G. $B+C+E$ & 170,000 & 99 & 11 & 6 & 3 & Medium & 30 & 0 & 2 & 1 & 0 \\
\hline \multicolumn{12}{|c|}{ ESV Marsh } \\
\hline A. No action & 0 & 97 & 87 & 8 & 5.7 & Medium & 15 & 10 & 3 & 1 & 0 \\
\hline $\begin{array}{l}\text { B. Trap mammalian predators, including rac- } \\
\text { coons, to increase nesting success of tidal } \\
\text { marsh birds }\end{array}$ & 50,000 & 98 & 87 & 8 & 6.1 & Medium & 15 & 10 & 3 & 1 & 0 \\
\hline C. Reduce deer population & 10,000 & 98 & 87 & 8 & 6.1 & Medium & 15 & 10 & 3 & 1 & 0 \\
\hline $\begin{array}{l}\text { D. Remove Wise Point Road and control } \\
\text { invasive plants }\end{array}$ & 100,000 & 98 & 87 & 8 & 5.9 & Medium & 15 & 10 & 3 & 1 & 24 \\
\hline $\begin{array}{l}\text { E. Remove water control structure on Wise } \\
\text { Point Road and control invasive plants }\end{array}$ & 10,000 & 98 & 87 & 8 & 5.9 & Medium & 15 & 10 & 3 & 1 & 6 \\
\hline $\begin{array}{l}\text { F. Remove Phragmites on Wise Point Road } \\
\text { and fringing marsh unit }\end{array}$ & 50,000 & 100 & 87 & 8 & 6 & Medium & 30 & 10 & 3 & 1 & 18 \\
\hline G. Reduce disturbance through refuge permits & 7,800 & 98 & 87 & 8 & 6 & Medium & 15 & 10 & 3 & 1 & 0 \\
\hline H. B+C & 60,000 & 98 & 87 & 8 & 6.6 & Medium & 15 & 10 & 3 & 1 & 0 \\
\hline I. Dig runnels by hand & 9,000 & 100 & 157 & 15 & 8.6 & Medium & 30 & 7 & 3 & 1 & 0 \\
\hline J. Dig runnels using machine & 26,000 & 100 & 124 & 13 & 8.9 & Medium & 30 & 0 & 3 & 1 & 0 \\
\hline
\end{tabular}




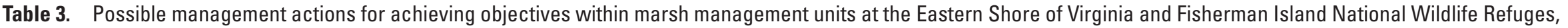
Virginia, estimated costs over 5 years, and predicted outcomes expressed relative to performance metrics.-Continued

[Potential management actions, costs, and predicted outcomes developed by workshop participants using expert judgement. Predicted consequences of management actions aided by influence diagrams (app. 1). $\%$, percent; ppt, parts per thousand]

\begin{tabular}{|c|c|c|c|c|c|c|c|c|c|c|c|}
\hline \multirow[b]{3}{*}{ Management action } & \multirow[b]{3}{*}{$\begin{array}{l}\text { Estimated } \\
\text { cost over } \\
5 \text { years } \\
\text { (dollars) }\end{array}$} & \multicolumn{10}{|c|}{ Performance metrics } \\
\hline & & \multirow[b]{2}{*}{$\begin{array}{l}\text { Native } \\
\text { vegetation } \\
\text { (\% cover) }\end{array}$} & \multicolumn{2}{|c|}{ Nekton } & \multirow[b]{2}{*}{$\begin{array}{c}\text { Tidal } \\
\text { marsh obli- } \\
\text { gate birds } \\
\text { (summed } \\
\text { number per } \\
\text { point) }\end{array}$} & \multirow[b]{2}{*}{$\begin{array}{l}\text { American } \\
\text { black } \\
\text { ducks } \\
\text { use }^{1}\end{array}$} & \multirow[b]{2}{*}{$\begin{array}{c}\text { Spider } \\
\text { density } \\
\text { (number } \\
\text { per } \\
\text { square } \\
\text { meter) }\end{array}$} & \multicolumn{2}{|c|}{ Hydrology } & \multirow{2}{*}{$\begin{array}{l}\text { Marsh } \\
\text { surface } \\
\text { elevation } \\
\text { change } \\
\text { relative to } \\
\text { sea-level } \\
\text { rise }^{3}\end{array}$} & \multirow[b]{2}{*}{$\begin{array}{c}\text { Herbicide } \\
\text { appli- } \\
\text { cation } \\
\text { (pints/ } \\
\text { year) }\end{array}$} \\
\hline & & & $\begin{array}{c}\text { Density } \\
\text { (number } \\
\text { of animals } \\
\text { per square } \\
\text { meter) }\end{array}$ & $\begin{array}{l}\text { Species } \\
\text { richness } \\
\text { (number) }\end{array}$ & & & & $\begin{array}{l}\text { Duration } \\
\text { of surface } \\
\text { flooding } \\
(\%)\end{array}$ & $\begin{array}{l}\text { Surface- } \\
\text { water } \\
\text { salinity }{ }^{2} \\
\text { (ppt) }\end{array}$ & & \\
\hline \multicolumn{12}{|c|}{ Raccoon } \\
\hline A. No action & 0 & 99 & 88 & 11 & 2 & Medium & 15 & 16.1 & 1 & 0 & 0 \\
\hline $\begin{array}{l}\text { B. Trap mammalian predators, including rac- } \\
\text { coons, to increase nesting success of tidal } \\
\text { marsh birds }\end{array}$ & 5,000 & 100 & 88 & 11 & 2.8 & Medium & 15 & 16.1 & 1 & 0 & 0 \\
\hline $\begin{array}{l}\text { C. Remove Phragmites on south end of marsh } \\
\text { unit }\end{array}$ & 10,000 & 100 & 88 & 11 & 2.8 & Medium & 15 & 16.1 & 1 & 0 & 24 \\
\hline D. Install living shoreline & $2,500,000$ & 100 & 120 & 11 & 2.8 & Medium & 30 & 16.1 & 1 & 1 & 0 \\
\hline \multicolumn{12}{|c|}{ Fisherman Island East Marsh } \\
\hline A. No action & 0 & 98 & 51 & 9 & 3.2 & Medium & 30 & 2.6 & 1 & 1 & 0 \\
\hline $\begin{array}{l}\text { B. Trap mammalian predators, including rac- } \\
\text { coons, to increase nesting success of tidal } \\
\text { marsh birds }\end{array}$ & 50,000 & 99 & 51 & 9 & 4 & Medium & 30 & 2.6 & 1 & 1 & 0 \\
\hline C. Reduce deer population & 10,000 & 99 & 51 & 9 & 4.1 & Medium & 30 & 2.6 & 1 & 1 & 0 \\
\hline D. Remove Phragmites on fringe of marsh unit & 150,000 & 100 & 51 & 9 & 3.6 & Medium & 30 & 2.6 & 1 & 1 & 360 \\
\hline E. Reduce disturbance through refuge permits & 7,800 & 100 & 51 & 9 & 3.6 & Medium & 30 & 2.6 & 1 & 1 & 0 \\
\hline \multicolumn{12}{|c|}{ Fisherman Island West Marsh } \\
\hline A. No action & 0 & 99 & 172 & 16 & 7.5 & Medium & 15 & 20 & 3 & 1 & 0 \\
\hline $\begin{array}{l}\text { B. Trap mammalian predators, including rac- } \\
\text { coons, to increase nesting success of tidal } \\
\text { marsh birds }\end{array}$ & 50,000 & 100 & 172 & 15 & 8.5 & Medium & 15 & 20 & 3 & 1 & 0 \\
\hline C. Reduce deer population & 10,000 & 100 & 172 & 15 & 8.9 & Medium & 15 & 20 & 3 & 1 & 0 \\
\hline $\begin{array}{l}\text { D. Install six road culverts or bridges and } \\
\text { control invasive plants }\end{array}$ & $6,000,000$ & 100 & 110 & 11 & 10 & Medium & 30 & 6 & 3 & 1 & 60 \\
\hline $\begin{array}{l}\text { E. Remove Phragmites on inland pond and } \\
\text { fringing marsh unit }\end{array}$ & 25,000 & 100 & 172 & 15 & 8.5 & Medium & 15 & 20 & 3 & 1 & 0 \\
\hline
\end{tabular}


Table 3. Possible management actions for achieving objectives within marsh management units at the Eastern Shore of Virginia and Fisherman Island National Wildlife Refuges, Virginia, estimated costs over 5 years, and predicted outcomes expressed relative to performance metrics.—Continued

[Potential management actions, costs, and predicted outcomes developed by workshop participants using expert judgement. Predicted consequences of management actions aided by influence diagrams (app. 1). $\%$, percent; ppt, parts per thousand]

\begin{tabular}{|c|c|c|c|c|c|c|c|c|c|c|c|}
\hline \multirow[b]{3}{*}{ Management action } & \multirow[b]{3}{*}{$\begin{array}{c}\text { Estimated } \\
\text { cost over } \\
5 \text { years } \\
\text { (dollars) }\end{array}$} & \multicolumn{10}{|c|}{ Performance metrics } \\
\hline & & \multirow[b]{2}{*}{$\begin{array}{c}\text { Native } \\
\text { vegetation } \\
\text { (\% cover) }\end{array}$} & \multicolumn{2}{|c|}{ Nekton } & \multirow[b]{2}{*}{$\begin{array}{c}\text { Tidal } \\
\text { marsh obli- } \\
\text { gate birds } \\
\text { (summed } \\
\text { number per } \\
\text { point) }\end{array}$} & \multirow[b]{2}{*}{$\begin{array}{c}\text { American } \\
\text { black } \\
\text { ducks } \\
\text { use }^{1}\end{array}$} & \multirow[b]{2}{*}{$\begin{array}{c}\text { Spider } \\
\text { density } \\
\text { (number } \\
\text { per } \\
\text { square } \\
\text { meter) }\end{array}$} & \multicolumn{2}{|c|}{ Hydrology } & \multirow[b]{2}{*}{$\begin{array}{l}\text { Marsh } \\
\text { surface } \\
\text { elevation } \\
\text { change } \\
\text { relative to } \\
\text { sea-level } \\
\text { rise }^{3}\end{array}$} & \multirow[b]{2}{*}{$\begin{array}{c}\text { Herbicide } \\
\text { appli- } \\
\text { cation } \\
\text { (pints/ } \\
\text { year) }\end{array}$} \\
\hline & & & $\begin{array}{c}\text { Density } \\
\text { (number } \\
\text { of animals } \\
\text { per square } \\
\text { meter) }\end{array}$ & $\begin{array}{l}\text { Species } \\
\text { richness } \\
\text { (number) }\end{array}$ & & & & $\begin{array}{c}\text { Duration } \\
\text { of surface } \\
\text { flooding }{ }^{2} \\
(\%)\end{array}$ & $\begin{array}{l}\text { Surface- } \\
\text { water } \\
\text { salinity }{ }^{2} \\
\text { (ppt) }\end{array}$ & & \\
\hline \multicolumn{12}{|c|}{ Fisherman Island West Marsh—Continued } \\
\hline $\begin{array}{l}\text { F. Install living shoreline to protect dunes and } \\
\text { prevent breaches into marsh }\end{array}$ & $5,000,000$ & 100 & 172 & 15 & 8.4 & Medium & 15 & 20 & 3 & 1 & 0 \\
\hline $\begin{array}{l}\text { G. Remove invasive plants in upland adjacent } \\
\text { to marsh }\end{array}$ & 10,000 & 100 & 172 & 15 & 8.4 & Medium & 15 & 20 & 3 & 1 & 0 \\
\hline H. Reduce disturbance through refuge permits & 7,800 & 100 & 172 & 15 & 8.5 & Medium & 15 & 20 & 3 & 1 & 0 \\
\hline I. Dig runnels by hand & 9,000 & 100 & 157 & 15 & 8.6 & Medium & 30 & 10 & 3 & 1 & 0 \\
\hline J. Dig runnels using machine & 26,000 & 100 & 124 & 13 & 8.9 & Medium & 30 & 8 & 3 & 1 & 0 \\
\hline K. D+J & $6,026,000$ & 100 & 87 & 9 & 9.3 & Medium & 30 & 0 & 3 & 1 & 60 \\
\hline
\end{tabular}

${ }^{1}$ Relative abundance for refuges during wintering waterfowl season.

${ }^{2}$ Measures absolute deviation from reference point representing ideal condition.

${ }^{3}$ Measures change relative to sea-level rise: 0 , lower than sea-level rise; 1 , above sea-level rise. 
Table 4. Normalized predicted outcomes and estimated total management benefits of possible management actions within six marsh management units at the Eastern Shore of Virginia and Fisherman Island National Wildlife Refuges, Virginia.

[Numeric table entries are weighted utilities, which were calculated as raw utilities multiplied by objective weights. Unitless raw utilities were derived from metric scores (table 3 ) using existing regional value functions (app. 2). Objective weights for individual metrics were calculated as the product of the weights on the branch of the objectives hierarchy leading to each metric (table 1). The total management benefit for each action is the sum of weighted utilities across all performance metrics]

\begin{tabular}{|c|c|c|c|c|c|c|c|c|c|c|c|}
\hline \multirow[b]{3}{*}{ Management action } & \multicolumn{10}{|c|}{ Performance metrics } & \multirow{3}{*}{$\begin{array}{c}\text { Total man- } \\
\text { agement } \\
\text { benefit }\end{array}$} \\
\hline & \multirow[b]{2}{*}{$\begin{array}{c}\text { Native } \\
\text { vegetation }\end{array}$} & \multicolumn{2}{|c|}{ Nekton } & \multirow{2}{*}{$\begin{array}{c}\text { Tidal } \\
\text { marsh } \\
\text { obligate } \\
\text { birds }\end{array}$} & \multirow[b]{2}{*}{$\begin{array}{l}\text { American } \\
\text { black } \\
\text { ducks }\end{array}$} & \multirow[b]{2}{*}{$\begin{array}{l}\text { Spider } \\
\text { density }\end{array}$} & \multicolumn{2}{|c|}{ Hydrology } & \multirow{2}{*}{$\begin{array}{c}\text { Marsh } \\
\text { surface } \\
\text { elevation } \\
\text { change }\end{array}$} & \multirow[b]{2}{*}{$\begin{array}{l}\text { Herbicide } \\
\text { application }\end{array}$} & \\
\hline & & Density & $\begin{array}{l}\text { Species } \\
\text { richness }\end{array}$ & & & & $\begin{array}{l}\text { Duration } \\
\text { of surface } \\
\text { flooding }\end{array}$ & $\begin{array}{l}\text { Surface- } \\
\text { water } \\
\text { salinity }\end{array}$ & & & \\
\hline \multicolumn{12}{|c|}{ Bull Marsh } \\
\hline A. No action & 0.119 & 0.028 & 0.020 & 0.035 & 0.075 & 0.090 & 0.108 & 0.110 & 0.220 & 0.060 & 0.865 \\
\hline $\begin{array}{l}\text { B. Remove road and control any resulting invasive } \\
\text { species }\end{array}$ & 0.120 & 0.032 & 0.028 & 0.055 & 0.075 & 0.090 & 0.110 & 0.110 & 0.220 & 0.059 & 0.899 \\
\hline $\begin{array}{l}\text { C. Breach road in four places and control any } \\
\text { resulting invasive species }\end{array}$ & 0.120 & 0.030 & 0.025 & 0.045 & 0.075 & 0.090 & 0.110 & 0.110 & 0.220 & 0.060 & 0.885 \\
\hline D. Lower road elevation & 0.120 & 0.029 & 0.023 & 0.045 & 0.075 & 0.090 & 0.110 & 0.110 & 0.220 & 0.060 & 0.882 \\
\hline $\begin{array}{l}\text { E. Remove Phragmites in western edge of unit } \\
\text { and field }\end{array}$ & 0.120 & 0.028 & 0.020 & 0.036 & 0.075 & 0.090 & 0.108 & 0.110 & 0.220 & 0.050 & 0.857 \\
\hline $\begin{array}{l}\text { F. Facilitate marsh migration by leveling berms } \\
\text { and ditches west of unit and controlling invasive } \\
\text { plants }\end{array}$ & 0.120 & 0.028 & 0.020 & 0.035 & 0.075 & 0.090 & 0.110 & 0.110 & 0.220 & 0.058 & 0.866 \\
\hline G. Create more pools and runnels & 0.120 & 0.034 & 0.023 & 0.035 & 0.075 & 0.045 & 0.108 & 0.110 & 0.220 & 0.060 & 0.829 \\
\hline $\begin{array}{l}\text { H. Trap mammalian predators, including Procyon } \\
\text { lotor (raccoon), to increase nesting success of } \\
\text { tidal marsh birds }\end{array}$ & 0.120 & 0.028 & 0.020 & 0.040 & 0.075 & 0.090 & 0.108 & 0.110 & 0.220 & 0.060 & 0.871 \\
\hline I. Reduce disturbance through refuge permits & 0.120 & 0.028 & 0.020 & 0.037 & 0.075 & 0.090 & 0.108 & 0.110 & 0.220 & 0.060 & 0.868 \\
\hline J. Acquire land and control Phragmites & 0.119 & 0.035 & 0.025 & 0.036 & 0.075 & 0.090 & 0.110 & 0.110 & 0.220 & 0.055 & 0.876 \\
\hline $\begin{array}{l}\text { K. Install three culverts in road and control inva- } \\
\text { sive plants }\end{array}$ & 0.120 & 0.030 & 0.025 & 0.045 & 0.075 & 0.090 & 0.110 & 0.110 & 0.220 & 0.060 & 0.884 \\
\hline L. $B+G$ & 0.120 & 0.039 & 0.031 & 0.057 & 0.075 & 0.045 & 0.108 & 0.110 & 0.220 & 0.060 & 0.865 \\
\hline \multicolumn{12}{|c|}{ Skidmore S } \\
\hline A. No action & 0.120 & 0.006 & 0.017 & 0.007 & 0.075 & 0.045 & 0.063 & 0.110 & 0.220 & 0.060 & 0.722 \\
\hline $\begin{array}{l}\text { B. Create islands for marsh bird nesting using } \\
\text { dredge spoils }\end{array}$ & 0.119 & 0.006 & 0.017 & 0.010 & 0.075 & 0.045 & 0.081 & 0.110 & 0.220 & 0.060 & 0.743 \\
\hline $\begin{array}{l}\text { C. Apply broad-scale sediment to marsh platform } \\
\text { to lower flood duration }\end{array}$ & 0.120 & 0.005 & 0.017 & 0.010 & 0.075 & 0.090 & 0.110 & 0.110 & 0.220 & 0.060 & 0.817 \\
\hline $\begin{array}{l}\text { D. Remove Phragmites on uplands and marsh } \\
\text { edge }\end{array}$ & 0.120 & 0.006 & 0.017 & 0.007 & 0.075 & 0.045 & 0.063 & 0.110 & 0.220 & 0.057 & 0.720 \\
\hline
\end{tabular}


Table 4. Normalized predicted outcomes and estimated total management benefits of possible management actions within six marsh management units at the Eastern Shore of Virginia and Fisherman Island National Wildlife Refuges, Virginia.-Continued

[Numeric table entries are weighted utilities, which were calculated as raw utilities multiplied by objective weights. Unitless raw utilities were derived from metric scores (table 3 ) using existing regional value functions (app. 2). Objective weights for individual metrics were calculated as the product of the weights on the branch of the objectives hierarchy leading to each metric (table 1). The total management benefit for each action is the sum of weighted utilities across all performance metrics]

\begin{tabular}{|c|c|c|c|c|c|c|c|c|c|c|c|}
\hline \multirow[b]{3}{*}{ Management action } & \multicolumn{10}{|c|}{ Performance metrics } & \multirow{3}{*}{$\begin{array}{c}\text { Total man- } \\
\text { agement } \\
\text { benefit }\end{array}$} \\
\hline & \multirow[b]{2}{*}{$\begin{array}{c}\text { Native } \\
\text { vegetation }\end{array}$} & \multicolumn{2}{|c|}{ Nekton } & \multirow{2}{*}{$\begin{array}{c}\text { Tidal } \\
\text { marsh } \\
\text { obligate } \\
\text { birds }\end{array}$} & \multirow[b]{2}{*}{$\begin{array}{c}\text { American } \\
\text { black } \\
\text { ducks }\end{array}$} & \multirow[b]{2}{*}{$\begin{array}{l}\text { Spider } \\
\text { density }\end{array}$} & \multicolumn{2}{|c|}{ Hydrology } & \multirow{2}{*}{$\begin{array}{c}\text { Marsh } \\
\text { surface } \\
\text { elevation } \\
\text { change }\end{array}$} & \multirow[b]{2}{*}{$\begin{array}{l}\text { Herbicide } \\
\text { application }\end{array}$} & \\
\hline & & Density & $\begin{array}{l}\text { Species } \\
\text { richness }\end{array}$ & & & & $\begin{array}{c}\text { Duration } \\
\text { of surface } \\
\text { flooding }\end{array}$ & $\begin{array}{c}\text { Surface- } \\
\text { water } \\
\text { salinity }\end{array}$ & & & \\
\hline \multicolumn{12}{|c|}{ Skidmore S-Continued } \\
\hline $\begin{array}{l}\text { E. Trap mammalian predators, including raccoons, } \\
\text { to increase nesting success of tidal marsh birds }\end{array}$ & 0.120 & 0.006 & 0.017 & 0.012 & 0.075 & 0.045 & 0.063 & 0.110 & 0.220 & 0.060 & 0.728 \\
\hline F. $B+E$ & 0.120 & 0.006 & 0.017 & 0.020 & 0.075 & 0.045 & 0.081 & 0.110 & 0.220 & 0.060 & 0.753 \\
\hline G. $\mathrm{B}+\mathrm{C}+\mathrm{E}$ & 0.120 & 0.005 & 0.017 & 0.030 & 0.075 & 0.090 & 0.110 & 0.110 & 0.220 & 0.060 & 0.837 \\
\hline \multicolumn{12}{|c|}{ ESV Marsh } \\
\hline A. No action & 0.119 & 0.031 & 0.023 & 0.057 & 0.075 & 0.045 & 0.110 & 0.110 & 0.220 & 0.060 & 0.849 \\
\hline $\begin{array}{l}\text { B. Trap mammalian predators, including raccoons, } \\
\text { to increase nesting success of tidal marsh birds }\end{array}$ & 0.119 & 0.031 & 0.023 & 0.061 & 0.075 & 0.045 & 0.110 & 0.110 & 0.220 & 0.060 & 0.854 \\
\hline C. Reduce deer population & 0.119 & 0.031 & 0.023 & 0.061 & 0.075 & 0.045 & 0.110 & 0.110 & 0.220 & 0.060 & 0.854 \\
\hline $\begin{array}{l}\text { D. Remove Wise Point Road and control invasive } \\
\text { plants }\end{array}$ & 0.119 & 0.031 & 0.023 & 0.059 & 0.075 & 0.045 & 0.110 & 0.110 & 0.220 & 0.056 & 0.848 \\
\hline $\begin{array}{l}\text { E. Remove water control structure on Wise Point } \\
\text { Road and control invasive plants }\end{array}$ & 0.119 & 0.031 & 0.023 & 0.059 & 0.075 & 0.045 & 0.110 & 0.110 & 0.220 & 0.059 & 0.851 \\
\hline $\begin{array}{l}\text { F. Remove Phragmites on Wise Point Road and } \\
\text { fringing marsh unit }\end{array}$ & 0.120 & 0.031 & 0.023 & 0.060 & 0.075 & 0.090 & 0.110 & 0.110 & 0.220 & 0.057 & 0.895 \\
\hline G. Reduce disturbance through refuge permits & 0.119 & 0.031 & 0.023 & 0.060 & 0.075 & 0.045 & 0.110 & 0.110 & 0.220 & 0.060 & 0.853 \\
\hline H. B $+\mathrm{C}$ & 0.119 & 0.031 & 0.023 & 0.066 & 0.075 & 0.045 & 0.110 & 0.110 & 0.220 & 0.060 & 0.859 \\
\hline I. Dig runnels by hand & 0.120 & 0.043 & 0.042 & 0.086 & 0.075 & 0.090 & 0.110 & 0.110 & 0.220 & 0.060 & 0.956 \\
\hline J. Dig runnels using machine & 0.120 & 0.038 & 0.037 & 0.089 & 0.075 & 0.090 & 0.110 & 0.110 & 0.220 & 0.060 & 0.949 \\
\hline \multicolumn{12}{|c|}{ Raccoon } \\
\hline A. No action & 0.120 & 0.031 & 0.031 & 0.020 & 0.075 & 0.045 & 0.088 & 0.110 & 0.000 & 0.060 & 0.579 \\
\hline $\begin{array}{l}\text { B. Trap mammalian predators, including raccoons, } \\
\text { to increase nesting success of tidal marsh birds }\end{array}$ & 0.120 & 0.031 & 0.031 & 0.028 & 0.075 & 0.045 & 0.088 & 0.110 & 0.000 & 0.060 & 0.588 \\
\hline C. Remove Phragmites on south end of marsh unit & 0.120 & 0.031 & 0.031 & 0.028 & 0.075 & 0.045 & 0.088 & 0.110 & 0.000 & 0.056 & 0.584 \\
\hline D. Install living shoreline & 0.120 & 0.038 & 0.031 & 0.028 & 0.075 & 0.090 & 0.088 & 0.110 & 0.220 & 0.060 & 0.859 \\
\hline
\end{tabular}


Table 4. Normalized predicted outcomes and estimated total management benefits of possible management actions within six marsh management units at the Eastern Shore of Virginia and Fisherman Island National Wildlife Refuges, Virginia.-Continued

Numeric table entries are weighted utilities, which were calculated as raw utilities multiplied by objective weights. Unitless raw utilities were derived from metric scores (table 3 ) using existing regional value functions (app. 2). Objective weights for individual metrics were calculated as the product of the weights on the branch of the objectives hierarchy leading to each metric (table 1). The total management benefit for each action is the sum of weighted utilities across all performance metrics]

\begin{tabular}{|c|c|c|c|c|c|c|c|c|c|c|c|}
\hline \multirow{3}{*}{ Management action } & \multicolumn{10}{|c|}{ Performance metrics } & \multirow{3}{*}{$\begin{array}{c}\text { Total man- } \\
\text { agement } \\
\text { benefit }\end{array}$} \\
\hline & \multirow[b]{2}{*}{$\begin{array}{c}\text { Native } \\
\text { vegetation }\end{array}$} & \multicolumn{2}{|c|}{ Nekton } & \multirow{2}{*}{$\begin{array}{c}\text { Tidal } \\
\text { marsh } \\
\text { obligate } \\
\text { birds }\end{array}$} & \multirow[b]{2}{*}{$\begin{array}{c}\text { American } \\
\text { black } \\
\text { ducks }\end{array}$} & \multirow[b]{2}{*}{$\begin{array}{l}\text { Spider } \\
\text { density }\end{array}$} & \multicolumn{2}{|c|}{ Hydrology } & \multirow{2}{*}{$\begin{array}{c}\text { Marsh } \\
\text { surface } \\
\text { elevation } \\
\text { change }\end{array}$} & \multirow[b]{2}{*}{$\begin{array}{l}\text { Herbicide } \\
\text { application }\end{array}$} & \\
\hline & & Density & $\begin{array}{l}\text { Species } \\
\text { richness }\end{array}$ & & & & $\begin{array}{l}\text { Duration } \\
\text { of surface } \\
\text { flooding }\end{array}$ & $\begin{array}{c}\text { Surface- } \\
\text { water } \\
\text { salinity }\end{array}$ & & & \\
\hline \multicolumn{12}{|c|}{ Fisherman Island East Marsh } \\
\hline A. No action & 0.119 & 0.021 & 0.025 & 0.032 & 0.075 & 0.090 & 0.110 & 0.110 & 0.220 & 0.060 & 0.862 \\
\hline $\begin{array}{l}\text { B. Trap mammalian predators, including raccoons, } \\
\text { to increase nesting success of tidal marsh birds }\end{array}$ & 0.120 & 0.021 & 0.025 & 0.040 & 0.075 & 0.090 & 0.110 & 0.110 & 0.220 & 0.060 & 0.871 \\
\hline C. Reduce deer population & 0.120 & 0.021 & 0.025 & 0.041 & 0.075 & 0.090 & 0.110 & 0.110 & 0.220 & 0.060 & 0.872 \\
\hline D. Remove Phragmites on fringe of marsh unit & 0.120 & 0.021 & 0.025 & 0.036 & 0.075 & 0.090 & 0.110 & 0.110 & 0.220 & 0.000 & 0.807 \\
\hline E. Reduce disturbance through refuge permits & 0.120 & 0.021 & 0.025 & 0.036 & 0.075 & 0.090 & 0.110 & 0.110 & 0.220 & 0.060 & 0.867 \\
\hline \multicolumn{12}{|c|}{ Fisherman Island West Marsh } \\
\hline A. No action & 0.120 & 0.045 & 0.045 & 0.075 & 0.075 & 0.045 & 0.073 & 0.110 & 0.220 & 0.060 & 0.868 \\
\hline $\begin{array}{l}\text { B. Trap mammalian predators, including raccoons, } \\
\text { to increase nesting success of tidal marsh birds }\end{array}$ & 0.120 & 0.045 & 0.042 & 0.085 & 0.075 & 0.045 & 0.073 & 0.110 & 0.220 & 0.060 & 0.876 \\
\hline C. Reduce deer population & 0.120 & 0.045 & 0.042 & 0.089 & 0.075 & 0.045 & 0.073 & 0.110 & 0.220 & 0.060 & 0.880 \\
\hline $\begin{array}{l}\text { D. Install six road culverts or bridges and control } \\
\text { invasive plants }\end{array}$ & 0.120 & 0.036 & 0.031 & 0.100 & 0.075 & 0.090 & 0.110 & 0.110 & 0.220 & 0.050 & 0.942 \\
\hline $\begin{array}{l}\text { E. Remove Phragmites on inland pond and fring- } \\
\text { ing marsh unit }\end{array}$ & 0.120 & 0.045 & 0.042 & 0.085 & 0.075 & 0.045 & 0.073 & 0.110 & 0.220 & 0.060 & 0.876 \\
\hline $\begin{array}{l}\text { F. Install living shoreline to protect dunes and } \\
\text { prevent breaches into marsh }\end{array}$ & 0.120 & 0.045 & 0.042 & 0.084 & 0.075 & 0.045 & 0.073 & 0.110 & 0.220 & 0.060 & 0.875 \\
\hline $\begin{array}{l}\text { G. Remove invasive plants in upland adjacent to } \\
\text { marsh }\end{array}$ & 0.120 & 0.045 & 0.042 & 0.084 & 0.075 & 0.045 & 0.073 & 0.110 & 0.220 & 0.060 & 0.875 \\
\hline H. Reduce disturbance through refuge permits & 0.120 & 0.045 & 0.042 & 0.085 & 0.075 & 0.045 & 0.073 & 0.110 & 0.220 & 0.060 & 0.876 \\
\hline I. Dig runnels by hand & 0.120 & 0.043 & 0.042 & 0.086 & 0.075 & 0.090 & 0.110 & 0.110 & 0.220 & 0.060 & 0.956 \\
\hline J. Dig runnels using machine & 0.120 & 0.038 & 0.037 & 0.089 & 0.075 & 0.090 & 0.110 & 0.110 & 0.220 & 0.060 & 0.949 \\
\hline K. D+J & 0.120 & 0.031 & 0.025 & 0.093 & 0.075 & 0.090 & 0.110 & 0.110 & 0.220 & 0.050 & 0.924 \\
\hline
\end{tabular}




\section{Results of Constrained Optimization}

Potential management actions identified to improve marsh integrity at the Eastern Shore of Virginia and Fisherman Island National Wildlife Refuges included trapping mammalian predators to increase the nesting success of tidal marsh birds; controlling Phragmites at the edges of the marsh management units; digging runnels, or shallow creeks, to enhance surface-water drainage; and restoring natural hydrology through breaching roads or installing culverts (table 3). For costs ranging from $\$ 0$ to $\$ 6.03$ million, the estimated management benefits for individual actions across all metrics, measured as weighted utilities, ranged from 0.579 (for implementing no action in the Raccoon management unit) to 0.956 (for digging runnels by hand in the ESV Marsh and Fisherman Island West Marsh management units), out of a maximum possible total management benefit of 1.0 (table 3, table 4). In two marsh management units (Raccoon, Fisherman Island West Marsh), the alternative with both the lowest management benefit and lowest cost was the "no action" alternative (management action A). However, in four marsh management units, implementing certain management actions (using herbicides to control invasive plants in Bull Marsh, Skidmore S, ESV Marsh, and Fisherman Island East Marsh; creating more pools and runnels in Bull Marsh) was predicted to yield a lower total management benefit than implementing no management action.

Constrained optimization was applied to identify the optimal management portfolios over 5 years for a range of total costs to the refuges' administration. As total cost increased from $\$ 0$ (no action in any unit) to approximately $\$ 3$ million, the total management benefit at the refuge scale increased from 4.746 to 5.379 (a 13-percent increase; table 5) out of a possible maximum of 6.0 (the maximum possible management benefit of 1.0 for any management action, summed across the six marsh management units). Further increases in the budget constraint yielded the same management portfolio (that is, portfolio 8, table 5). Graphical analysis showed a fairly consistent increase in management benefit as costs increased to $\$ 143,000$ (fig. 3, portfolio 5). Portfolio 5 represented the turning point in the cost-benefit plot. As expenditures increased beyond the cost of portfolio 5, total management benefit continued to increase but at a lower rate, yielding diminishing returns on investment (fig. 3).

The potential management actions selected within the set of portfolios that yielded the greatest total management benefit per unit cost (table 5, portfolios 2 through 5) largely differed across the marsh management units. In two marsh management units (ESV Marsh and Fisherman Island West Marsh), these portfolios always included hand-dug runnels as the optimum management action. In the other marsh management units these portfolios included breaching a road to restore natural hydrology (Bull Marsh); creating islands for marshnesting birds or spreading sediment to raise marsh elevation (Skidmore S); trapping predators to enhance nest success of tidal marsh obligate birds (Raccoon); and reducing the abundance of deer to minimize their effects on marsh vegetation (Fisherman Island East Marsh). Some potential management actions were never included in the portfolios yielding the greatest benefit per cost. For example, although applying herbicide to remove Phragmites was identified to improve the integrity of all marsh management units, this action was never selected. Similarly, reducing disturbance to the marsh by adding restrictions to refuge permits was suggested as a possible

Table 5. Actions included in various management portfolios to maximize the total management benefits subject to increasing cost constraints at the Eastern Shore of Virginia and Fisherman Island National Wildlife Refuges, Virginia.

[Letter designations for actions refer to specific actions and are listed in table 3 and table 4. Portfolios represent the combination of potential actions, one per marsh management unit, that maximized the total management benefit across all units, subject to a refuge-wide cost constraint. The management actions constituting individual portfolios were selected using constrained optimization. The total cost represents the sum of costs estimated for each action included in the portfolio. The maximum possible total management benefit for the combined refuges is 6.0 , derived as the maximum possible total management benefit of 1.0 for any management action within one management unit, summed across six units.]

\begin{tabular}{|c|c|c|c|c|c|c|c|c|}
\hline \multirow[b]{2}{*}{ Portfolio } & \multicolumn{6}{|c|}{ Marsh management unit } & \multirow[b]{2}{*}{$\begin{array}{l}\text { Total cost } \\
\text { (dollars) }\end{array}$} & \multirow[b]{2}{*}{$\begin{array}{l}\text { Total manage- } \\
\text { ment benefit }\end{array}$} \\
\hline & Bull Marsh & Skidmore S & ESV Marsh & Raccoon & $\begin{array}{c}\text { Fisherman } \\
\text { Island East } \\
\text { Marsh }\end{array}$ & $\begin{array}{c}\text { Fisherman } \\
\text { Island West } \\
\text { Marsh }\end{array}$ & & \\
\hline 1 & $\mathrm{~A}$ & A & A & $\mathrm{A}$ & $\mathrm{A}$ & $\mathrm{A}$ & 0 & 4.746 \\
\hline 2 & A & A & I & $\mathrm{B}$ & A & I & 23,000 & 4.950 \\
\hline 3 & $\mathrm{C}$ & A & I & A & $\mathrm{C}$ & I & 48,000 & 4.971 \\
\hline 5 & $\mathrm{C}$ & $\mathrm{C}$ & I & $\mathrm{B}$ & $\mathrm{C}$ & I & 143,000 & 5.074 \\
\hline 6 & $\mathrm{C}$ & $\mathrm{G}$ & I & $\mathrm{B}$ & $\mathrm{C}$ & I & 223,000 & 5.094 \\
\hline 7 & $\mathrm{~B}$ & G & I & $\mathrm{B}$ & $\mathrm{C}$ & I & 453,000 & 5.108 \\
\hline 8 & $\mathrm{~B}$ & G & I & $\mathrm{D}$ & $\mathrm{C}$ & I & $2,948,000$ & 5.379 \\
\hline
\end{tabular}




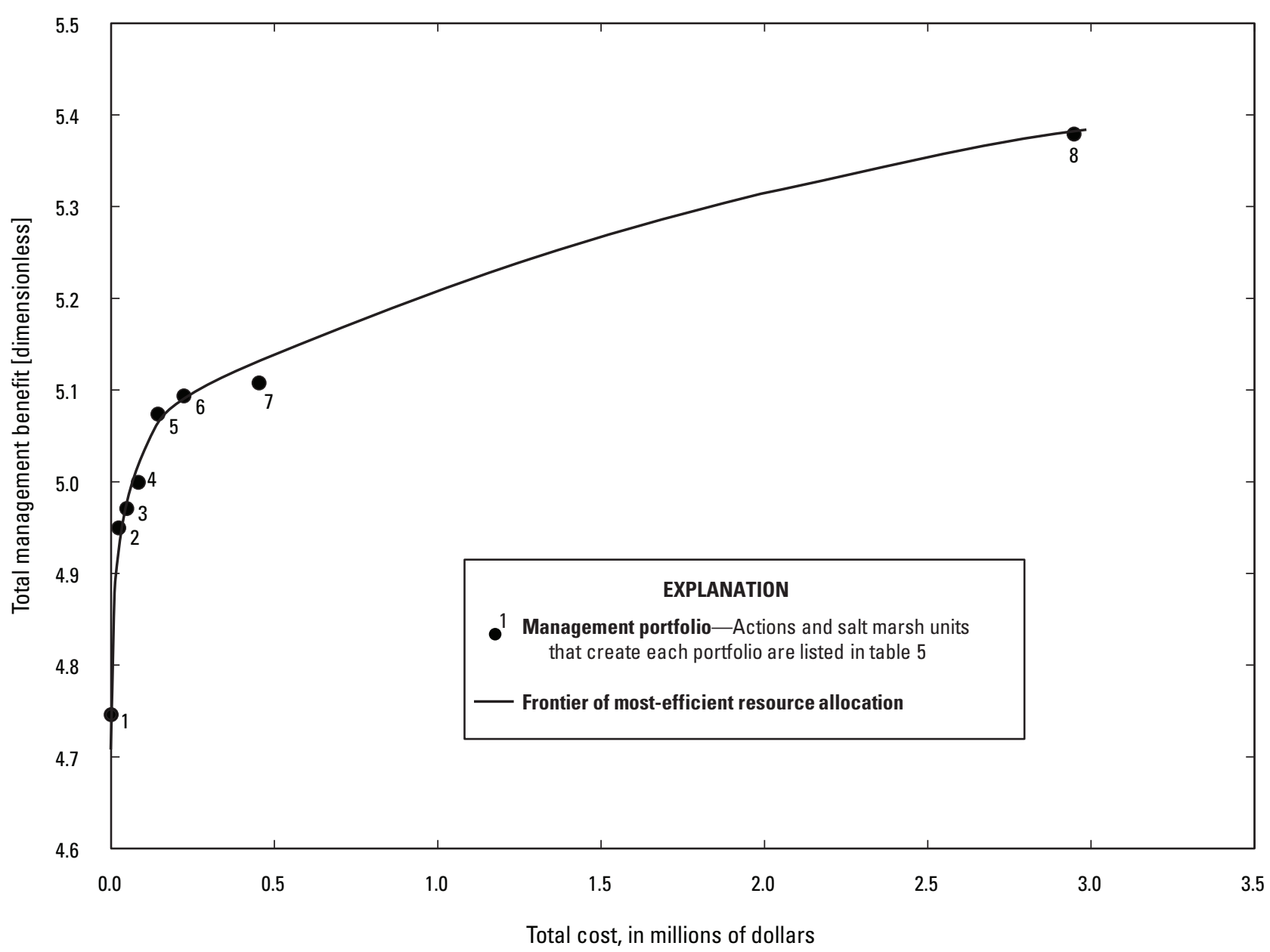

Figure 3. Graph showing predicted total management benefit of various portfolios, expressed as weighted utilities, relative to total cost at the Eastern Shore of Virginia and Fisherman Island National Wildlife Refuges in Virginia. Each portfolio (dot with number) represents a combination of six management actions, one per marsh management unit, as identified in table 5 . The line represents the efficient frontier for resource allocation.

action in four marsh management units, but predicted benefits (increases in numbers of tidal marsh obligate birds) were low and this action was never included in an optimal portfolio.

Examination of the refuge-scale metric responses to actions included in portfolio 5 , which is the turning point in the cost-benefit plot (fig. 3), revealed how implementation could affect specific management objectives. The actions included were predicted to achieve gains in the overall management benefits derived from density of spiders (as an indicator of trophic health), number of tidal marsh obligate breeding birds, species richness of nekton, and reduced flooding duration (fig. 4). Ecologically, the combination of actions in portfolio 5 may result in the following average changes (derived as the relative difference between the predicted metric scores for the actions implemented in portfolio 5 and the "no-action" alternative within each marsh management unit, averaged across all units; table 3): a 77-percent increase in tidal marsh obligate bird counts, a 39-percent decrease in the deviation of surface flooding from the ideal reference condition, an 18-percent increase in nekton species richness, and a 50-percent increase in spider density. The management benefits predicted for portfolios 2 through 4 , at total costs up to $\$ 83,000$, were derived primarily from expected improvements in surface-water drainage, and presumed increases in densities of spiders and numbers of tidal marsh obligate birds (table 3, table 4). 


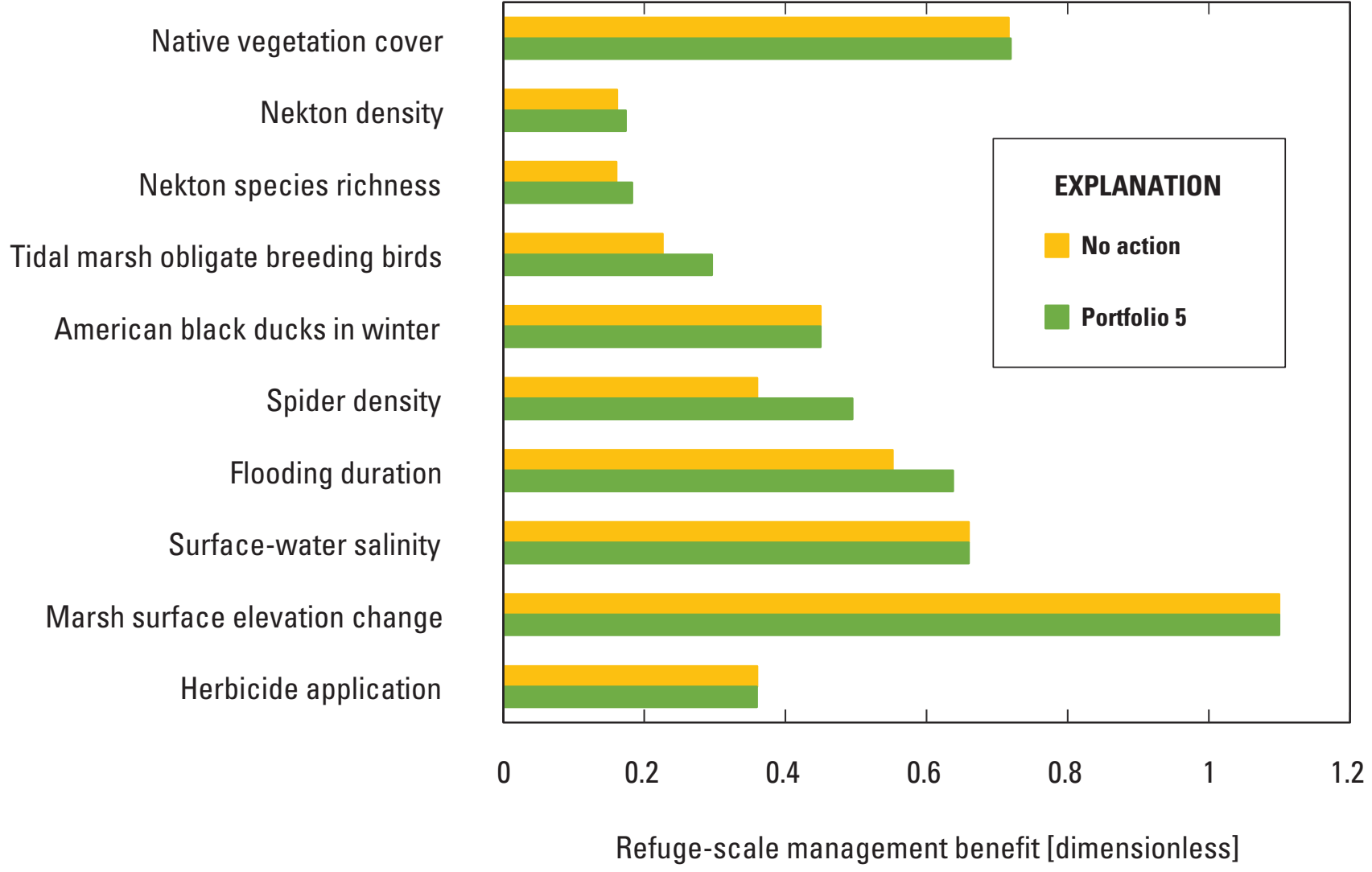

Figure 4. Bar chart showing predicted management benefit at the refuge scale for individual performance metrics, expressed as weighted utilities, resulting from implementation of the management actions included in portfolio 5, in comparison to the management benefit from the baseline "no-action" portfolio, at the Eastern Shore of Virginia and Fisherman Island National Wildlife Refuges in Virginia. The actions included in each portfolio are listed in table 5.

\section{Considerations for Optimizing Salt Marsh Management}

A regional structured decision-making framework for salt marshes on NWRs in the northeastern United States was applied by the USGS, in cooperation with the FWS, to develop a tool for optimizing management decisions at the Eastern Shore of Virginia and Fisherman Island National Wildlife Refuges. Use of the existing regional framework and a rapid-prototyping approach permitted NWR biologists and managers, FWS regional authorities, and research scientists to construct a decision model for the refuges within the confines of a 1.5-day workshop. This preliminary prototype provides a local framework for decision making while revealing information needs for future iterations. Insights from this process may also be useful to inform future habitat management planning at the refuges.

The suite of potential management actions and predicted outcomes included in this prototype (table 3 ) were based on current understanding of the Eastern Shore of Virginia and Fisherman Island National Wildlife Refuge salt marshes and hypothesized process-response pathways (app. 1). Tidal flooding is the predominant physical control on the structure and function of salt marsh ecosystems (Pennings and Bertness, 2001), and there is widespread scientific effort to elucidate how salt marshes may respond to accelerating rates of sea-level rise and management strategies to enhance their sustainability (Kirwan and Megonigal, 2013; Roman, 2017). Management interventions frequently proposed to mitigate effects of increased inundation on coastal marsh soils include increasing marsh drainage and raising marsh elevation (Wigand and others, 2017). In this prototype, digging runnels by hand to improve drainage of the marsh platform (in ESV Marsh and Fisherman Island West Marsh management units) and broad-scale deposition of sediments to raise marsh elevation (Skidmore S management unit) were predicted to yield similar increases in total management benefit, but sediment deposition was an order of magnitude more costly (that is, $\$ 90,000$ compared to $\$ 9,000$; table 3 , table 4 ). Multiple, interacting factors influence the long-term success of restoration actions in prolonging marsh integrity and improving marsh resilience, and ecosystem responses may depend on site-specific factors (Roman, 2017; Raposa and others, 2018; Perry and others, in press). Future iterations of this decision model can incorporate improved understanding 
of both implementation costs and marsh responses to management actions. In addition, during construction of the regional decision model, lack of widely available data on rates of vertical marsh growth led to the adoption of a very coarse scale of measurement for change in marsh surface elevation relative to sea-level rise (table 1). In 2012, surface elevation tables (Lynch and others, 2015) were installed in each marsh management unit to obtain high-resolution measurements of change in marsh surface elevation. Incorporating this information into subsequent iterations of this structured decisionmaking framework would likely improve predictions related to the potential for marsh surface elevation to keep pace with sea-level rise.

Results of constrained optimizations (table 5) based on the objectives, management actions, and predicted outcomes included in this prototype identified four areas in which to improve the utility of the prototype for refuge decision making. First, although minimizing the spread of Phragmites is a management concern at the Eastern Shore of Virginia and Fisherman Island National Wildlife Refuges, removing Phragmites through use of herbicides was not selected for any optimal portfolio. The transparency of the structured decisionmaking framework reveals the tradeoffs associated with applying herbicide to reduce the spread of invasive plants. In most instances, controlling invasive plants was predicted to increase the percent cover of native vegetation and the abundance of tidal marsh obligate birds (table 3 ) and the management benefits associated with achieving these specific objectives (table 4). However, spraying herbicide, which is a potential environmental contaminant, had direct negative consequences on the objective to minimize herbicide use and often resulted in a net decrease in the total management benefit (table 4). Thus, the benefits associated with use of herbicide to reduce invasive plants may not offset the negative value of applying environmental contaminants. These results emphasize the importance that refuge managers have placed on reducing spread of invasive plants through various methods, including mechanical control and prescribed burning (Denmon, 2018). This prototype could be adapted to allow managers to evaluate the relative expected benefits and detriments of chemical and other control methods.

Second, the habitat management plan for the Eastern Shore of Virginia and Fisherman Island National Wildlife Refuges identifies seaside sparrows as a priority resource of concern in salt marsh habitat (Denmon, 2018). These birds nest primarily in smooth cordgrass cover characteristic of the low marsh zone, where the tall vegetation allows relatively high nest placement that minimizes risks of tidal inundation (Gjerdrum and others, 2005). However, nest predation increases with nest height, and recent studies suggest that as sea-level rise increases the vulnerability of nests to tidal flooding, predator control may be required to maintain viability of seaside sparrow populations (Hunter, 2017; Roberts and others, 2019). In this prototype, trapping predators to enhance nest success of tidal marsh birds was predicted to improve the total management benefit in all marsh management units; however, this action was included in an optimal management portfolio for the Raccoon management unit only, where the estimated cost of implementation was lowest. Therefore, managers may want to explore alternative approaches to reduce nest predation, such as fencing around individual nests (Post and Greenlaw, 1989), in future prototypes.

Third, although loss of marsh area through shoreline erosion is a concern in the Raccoon and Fisherman Island West Marsh management units, reducing wave action through construction of living shorelines (plants or other natural elements for shoreline stabilization) was excluded from all but the costliest optimal portfolio. Living shorelines may be cost prohibitive at these refuges. Additionally, deconstructing the objective of maintaining the extent of the marsh platform into subordinate objectives and performance metrics related to both horizontal and vertical gains and losses of marsh substrate may help focus decision making on erosion of marsh edges as a driver of marsh area.

Finally, the constrained optimizations analyzed in this report were based on estimations of management costs. As salt marsh management is undertaken around the northeast region, a detailed list of actual expenses can be compiled, including staff time for project planning as well as materials, equipment, contracts, and staff time for implementation. For example, Iacona and others (2018) recommended a standardized approach for summarizing and reporting financial costs of management interventions. This could allow future iterations of this decision model to include more accurate cost estimates, while contributing information to help others identify costeffective salt marsh management techniques.

The prototype model for the Eastern Shore of Virginia and Fisherman Island National Wildlife Refuges provides a useful tool for decision making that can be updated in the future with new data and information. The spatial and temporal variability inherent in parameter estimates were not quantified during rapid prototyping. Previously, preliminary sensitivity analysis revealed little effect of incorporating ecological variation in abundance of marsh-obligate breeding birds on the optimal solutions for Prime Hook National Wildlife Refuge (Neckles and others, 2015). This lends confidence to use of this framework for decision making; however, including probability distributions for each performance metric in the decision model could be a high priority for future prototypes. Future monitoring of salt marsh integrity performance metrics will be useful to refine baseline parameter estimates and to determine the background rate of change in the absence of management actions; feedback from measured responses to management actions around the region will help reduce uncertainties surrounding management predictions. The structured decision-making framework applied here to the Eastern Shore of Virginia and Fisherman Island National Wildlife Refuges is based on a hierarchy of regional objectives and regional value functions relating performance metrics to perceived management benefits. It will be important to ensure that subsequent iterations reflect evolving management objectives and desired outcomes. Elements of the decision model could be further 
adapted, for example, through differential weighting of objectives or altered value functions, to reflect specific, local management goals and mandates. Future optimization analyses that use this framework could also incorporate additional constraints on action selection, such as ensuring that particular actions within individual marsh management units are included in optimal management portfolios, to further tailor the model to refuge-specific needs.

\section{References Cited}

Blomquist, S.M., Johnson, T.D., Smith, D.R., Call, G.P., Miller, B.N., Thurman, W.M., McFadden, J.E., Parkin, M.J., and Boomer, G.S., 2010, Structured decision-making and rapid prototyping to plan a management response to an invasive species: Journal of Fish and Wildlife Management, v. 1, no. 1, p. 19-32. [Also available at https://doi.org/ 10.3996/JFWM-025.]

Conroy, M.J., and Peterson, J.T., 2013, Decision making in natural resource management-A structured, adaptive approach: Chichester, United Kingdom, John Wiley and Sons, Ltd., 456 p. [Also available at https://doi.org/10.1002/ 9781118506196.]

Cowardin, L.M., Carter, V., Golet, F.C., and LaRoe, E.T., 1979, Classification of wetlands and deepwater habits of the United States: U.S. Fish and Wildlife Service FWS/ OBS-79/31, 131 p., accessed November 12, 2018, at https://www.fws.gov/wetlands/Documents/Classification-ofWetlands-and-Deepwater-Habitats-of-the-United-States.pdf.

Denmon, P., 2018, Eastern Shore of Virginia and Fisherman Island National Wildlife Refuges-Habitat management plan: U.S. Fish and Wildlife Service, [variously paged], accessed February 22, 2021, at https://ecos.fws.gov/ ServCat/Reference/Profile/111296.

Garrard, G.E., Rumpff, L., Runge, M.C., and Converse, S.J., 2017, Rapid prototyping for decision structuring An efficient approach to conservation decision analysis, in Bunnefeld, N., Nicholson, E., and Milner-Gulland, E.J., eds., Decision-making in conservation and natural resource management: Cambridge, United Kingdom, Cambridge University Press, p. 46-64. [Also available at https://doi.org/10.1017/9781316135938.003.]

Gedan, K.B., Altieri, A.H., and Bertness, M.D., 2011, Uncertain future of New England salt marshes: Marine Ecology Progress Series, v. 434, p. 229-237. [Also available at https://doi.org/10.3354/meps09084.]
Gedan, K.B., Silliman, B.R., and Bertness, M.D., 2009, Centuries of human-driven change in salt marsh ecosystems: Annual Review of Marine Science, v. 1, no. 1, p. 117-141. [Also available at https://doi.org/10.1146/ annurev.marine.010908.163930.]

Gjerdrum, C., Elphick, C.S., and Rubega, M., 2005, Nest site selection and nesting success in saltmarsh breeding sparrows - The importance of nest habitat, timing, and study site differences: The Condor, v. 107, no. 4, p. 849-862. [Also available at https://doi.org/10.1093/ condor/107.4.849.]

Gregory, R., Failing, L., Harstone, M., Long, G., McDaniels, T., and Ohlson, D., 2012, Structured decision making-A practical guide to environmental management choices: Chichester, United Kingdom, John Wiley and Sons, Ltd., 299 p. [Also available at https://doi.org/10.1002/ 9781444398557.]

Gregory, R.S., and Keeney, R.L., 2002, Making smarter environmental management decisions: Journal of the American Water Resources Association, v. 38, no. 6, p. 1601-1612. [Also available at https://doi.org/10.1111/ j.1752-1688.2002.tb04367.x.]

Hammond, J.S., Keeney, R.L., and Raiffa, H., 1999, Smart choices-A practical guide to making better life decisions: Boston, Harvard Business School Press, 242 p.

Hunter, E.A., 2017, How will sea-level rise affect threats to nesting success for seaside sparrows?: The Condor, v. 119, no. 3, p. 459-468. [Also available at https://doi.org/10.1650/ condor-17-11.1.]

Iacona, G.D., Sutherland, W.J., Mappin, B., Adams, V.M., Armsworth, P.R., Coleshaw, T., Cook, C., Craigie, I., Dicks, L.V., Fitzsimons, J.A., McGowan, J., Plumptre, A.J., Polak, T., Pullin, A.S., Ringma, J., Rushworth, I., Santangeli, A., Stewart, A., Tulloch, A., Walsh, J.C., and Possingham, H.P., 2018, Standardized reporting of the costs of management interventions for biodiversity conservation: Conservation Biology, v. 32, no. 5, p. 979-988. [Also available at https://doi.org/10.1111/cobi.13195.]

Keeney, R.L., and Raiffa, H., 1993, Decisions with multiple objectives-Preferences and value tradeoffs: Cambridge, United Kingdom, Cambridge University Press, 569 p. [Also available at https://doi.org/10.1017/CBO9781139174084.]

Kirkwood, C.W., 1997, Strategic decision makingMultiobjective decision analysis with spreadsheets: Belmont, Calif., Duxbury Press, 345 p.

Kirwan, M.L., and Megonigal, J.P., 2013, Tidal wetland stability in the face of human impacts and sea-level rise: Nature, v. 504, no. 7478 , p. 53-60. [Also available at https://doi.org/10.1038/nature12856.] 
Lynch, J.C., Hensel, P., and Cahoon, D.R., 2015, The surface elevation table and marker horizon technique-A protocol for monitoring wetland elevation dynamics: National Park Service Natural Resource Report NPS/NCBN/NRR 2015/1078, [variously paged], accessed August 24, 2018, at https://irma.nps.gov/DataStore/DownloadFile/531681.

Multi-Resolution Land Characteristics Consortium, 2020, NLCD 2011 land cover (CONUS): Multi-Resolution Land Characteristics Consortium database, accessed March 1, 2021, at https://www.mrlc.gov/data.

Neckles, H.A., Guntenspergen, G.R., Shriver, W.G., Danz, N.P., Wiest, W.A., Nagel, J.L., and Olker, J.H., 2013, Identification of metrics to monitor salt marsh integrity on national wildlife refuges in relation to conservation and management objectives: U.S. Fish and Wildlife Service, prepared by U.S. Geological Survey, 226 p., accessed May 1, 2018, at https://ecos.fws.gov/ServCat/Reference/ Profile/37795.

Neckles, H.A., Lyons, J.E., Guntenspergen, G.R., Shriver, W.G., and Adamowicz, S.C., 2015, Use of structured decision making to identify monitoring variables and management priorities for salt marsh ecosystems: Estuaries and Coasts, v. 38, no. 4, p. 1215-1232. [Also available at https://doi.org/10.1007/s12237-014-9822-5.]

Pennings, S.C., and Bertness, M.D., 2001, Salt marsh communities, in Bertness, M.D., Gaines, S.D., and Hay, M.E., eds., Marine community ecology: Sunderland, Mass., Sinauer Associates, p. 289-316.

Perry, D.C., Ferguson, W., and Thornber, C.S., [in press], Salt marsh climate adaptation-Using runnels to adapt to accelerating sea level rise within a drowning New England salt marsh: Restoration Ecology, article e13466. [Available at https://doi.org/10.1111/rec.13466.]

Post, W., and Greenlaw, J.S., 1989, Metal barriers protect nearground nests from predators: Journal of Field Ornithology, v. 60 , no. 1, p. 102-103. [Also available at https://sor a.unm.edu/sites/default/files/journals/jfo/v060n01/p0102p0103.pdf.]

Raposa, K.B., Lerberg, S., Cornu, C., Fear, J., Garfield, N., Peter, C., Weber, R.L.J., Moore, G., Burdick, D., and Dionne, M., 2018, Evaluating tidal wetland restoration performance using National Estuarine Research Reserve System reference sites and the restoration performance index (RPI): Estuaries and Coasts, v. 41, no. 1, p. 36-51. [Also available at https://doi.org/10.1007/s12237017-0220-7.]
Roberts, S.G., Longenecker, R.A., Etterson, M.A., Elphick, C.S., Olsen, B.J., and Shriver, W.G., 2019, Preventing local extinctions of tidal marsh endemic seaside sparrows and saltmarsh sparrows in eastern North America: The Condor, v. 121, no. 2, article duy024, p. 1-14. [Also available at https://doi.org/10.1093/condor/duy024.]

Roman, C.T., 2017, Salt marsh sustainability_Challenges during an uncertain future: Estuaries and Coasts, v. 40, no. 3, p. 711-716. [Also available at https://doi.org/10.1007/ s12237-016-0149-2.]

Steinkamp, M., 2008. New England/mid-Atlantic coast bird conservation (BCR 30) implementation plan: Laurel, Md., Atlantic Coast Joint Venture, 251 p., accessed August 15, 2018, at https://www.acjv.org/BCR_30/BCR30_June_23_ 2008 final.pdf.

U.S. Fish and Wildlife Service [FWS], 2004, Eastern Shore of Virginia and Fisherman Island National Wildlife RefugesComprehensive conservation plan: U.S. Fish and Wildlife Service, [variously paged], accessed February 22, 2021, at https://www.fws.gov/refuge/Eastern_Shore_of_Virginia/ what_we_do/finalccp.html.

U.S. Fish and Wildlife Service [FWS], 2016, Salt marsh integrity and Hurricane Sandy vegetation, bird and nekton data: U.S. Fish and Wildlife Service database, https://ecos.fws.gov/ServCat/Reference/Profile/121918.

U.S. North American Bird Conservation Initiative, 2020, Bird conservation regions: U.S. North American Bird Conservation Initiative web page, accessed July 6, 2020, at https://nabci-us.org/resources/bird-conservation-regions/.

Wigand, C., Ardito, T., Chaffee, C., Ferguson, W., Paton, S., Raposa, K., Vandemoer, C., and Watson, E., 2017, A climate change adaptation strategy for management of coastal marsh systems: Estuaries and Coasts, v. 40, no. 3, p. 682-693. [Also available at https://doi.org/10.1007/ s12237-015-0003-y.] 



\section{Appendix 1. Regional Influence Diagrams}

The influence diagrams (following the style of prototype diagrams in Neckles and others, 2015) in this appendix (figs. 1.1-1.8) relate possible management strategies to performance metrics. Shapes represent elements of decisions, as follows: rectangles for actions, rectangles with rounded corners for deterministic factors, ovals for stochastic events, and hexagons for consequences expressed as a performance metric.

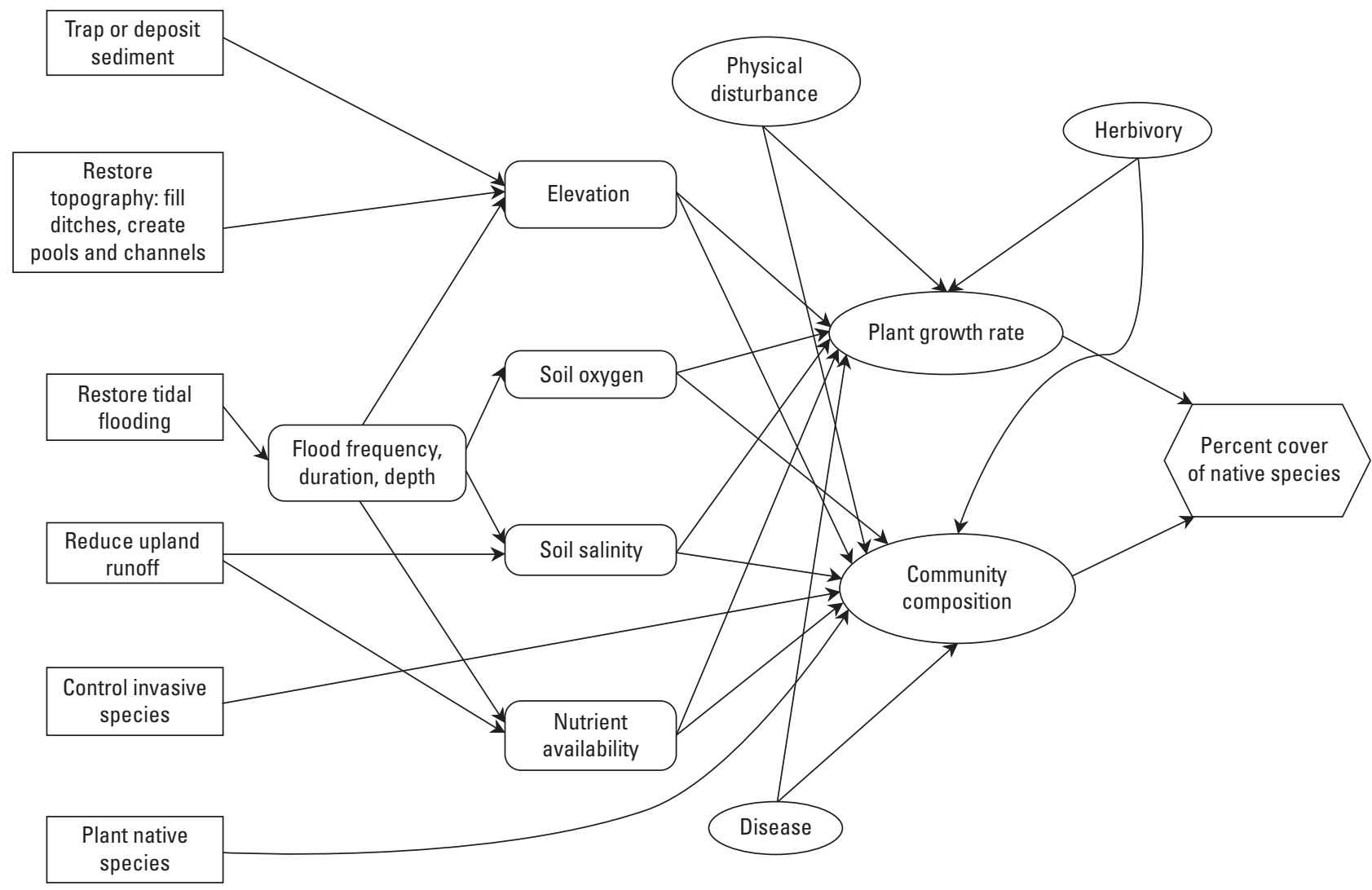

Figure 1.1. Influence diagram used to estimate percent cover of native vegetation in response to implementing certain management actions. 


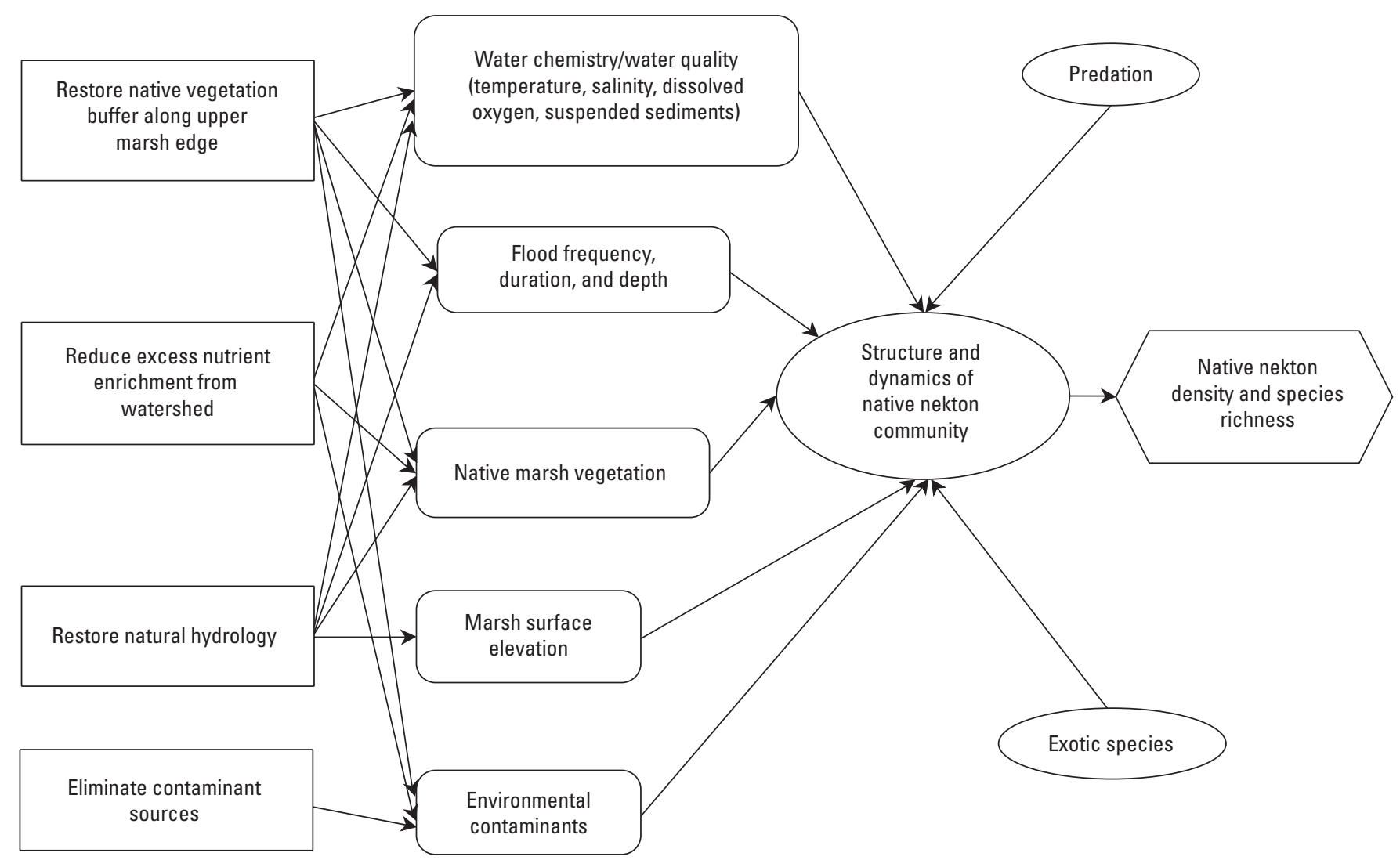

Figure 1.2. Influence diagram used to estimate nekton density and species richness in response to implementing certain management actions. 


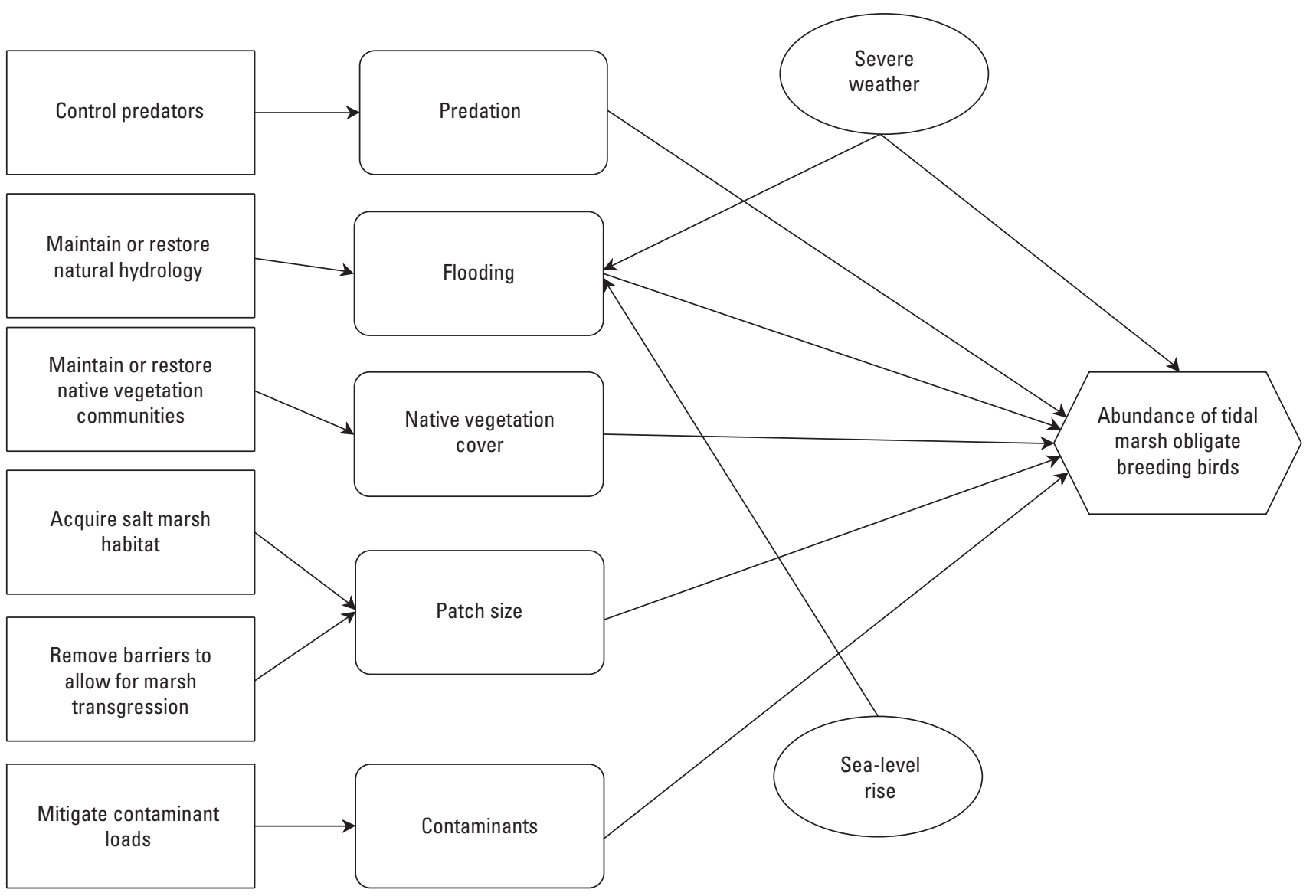

Figure 1.3. Influence diagram used to estimate abundance of tidal marsh obligate breeding birds in response to implementing certain management actions. 


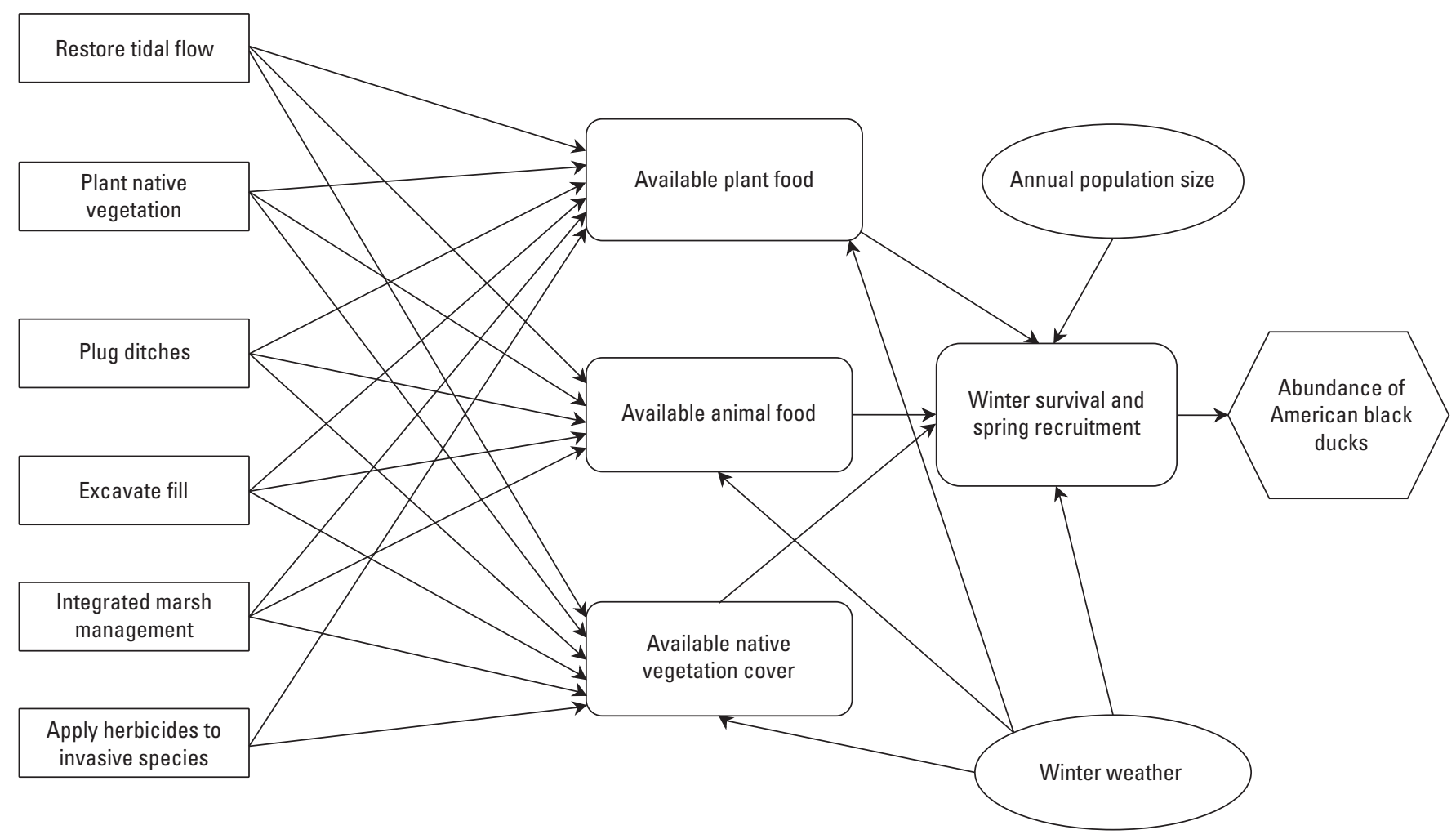

Figure 1.4. Influence diagram used to estimate abundance of American black ducks in winter, as indicator species for nonbreeding wetland birds, in response to implementing certain management actions. 


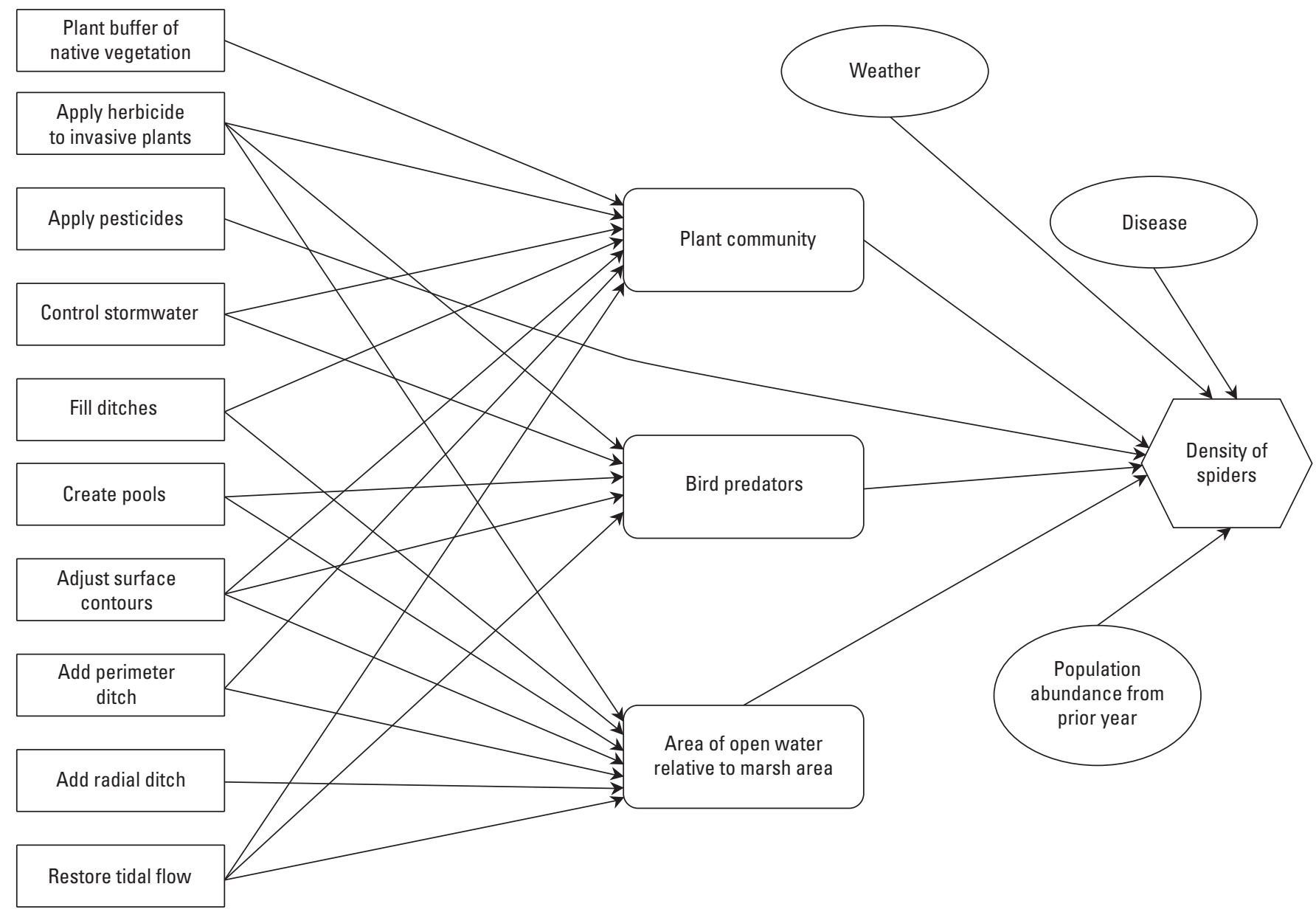

Figure 1.5. Influence diagram used to estimate density of spiders, as indic ator of trophic health, in response to implementing certain management actions. 


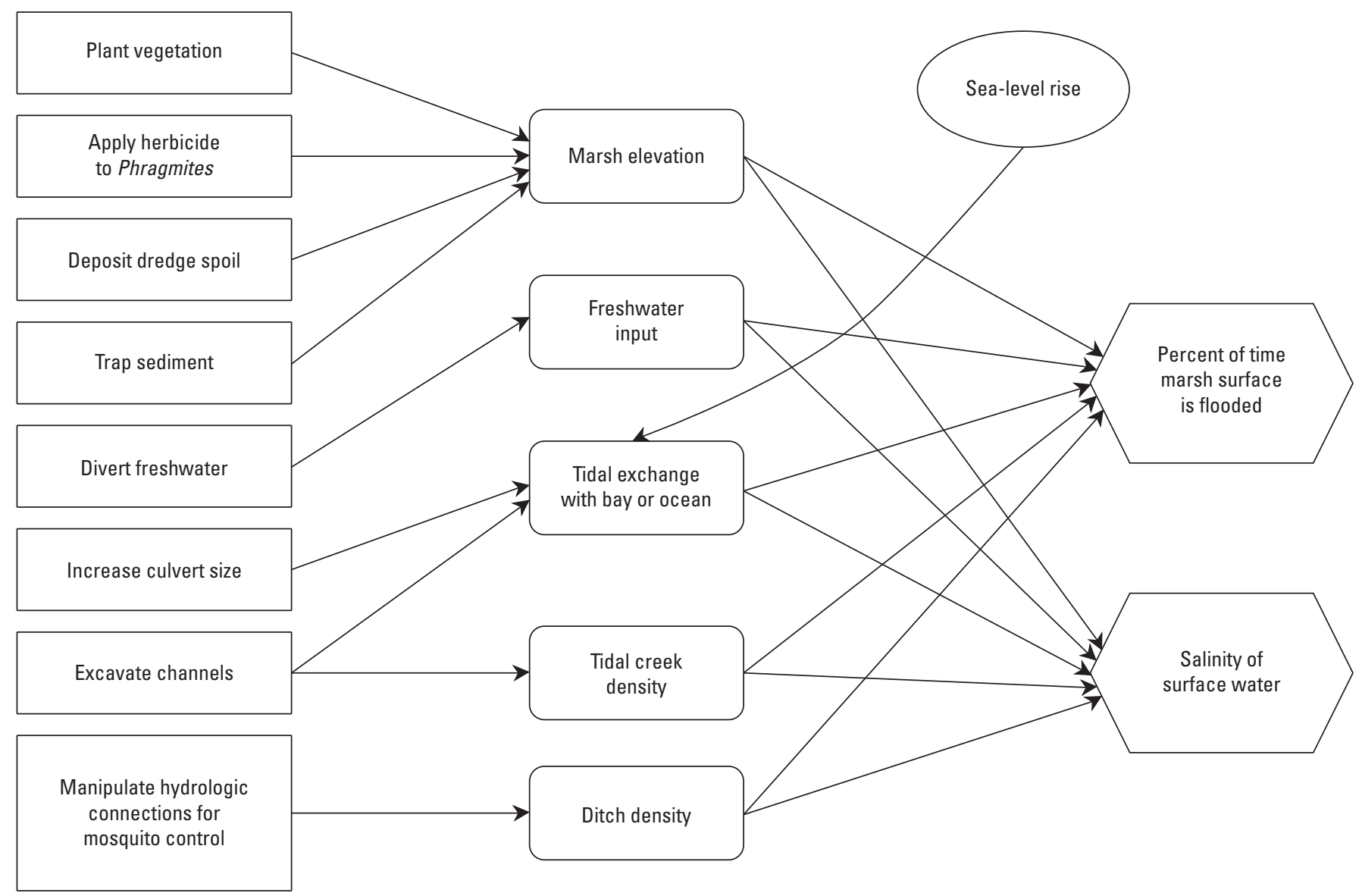

Figure 1.6. Influence diagram used to estimate percent of time marsh surface is flooded and salinity of marsh surface water in response to implementing certain management actions. 


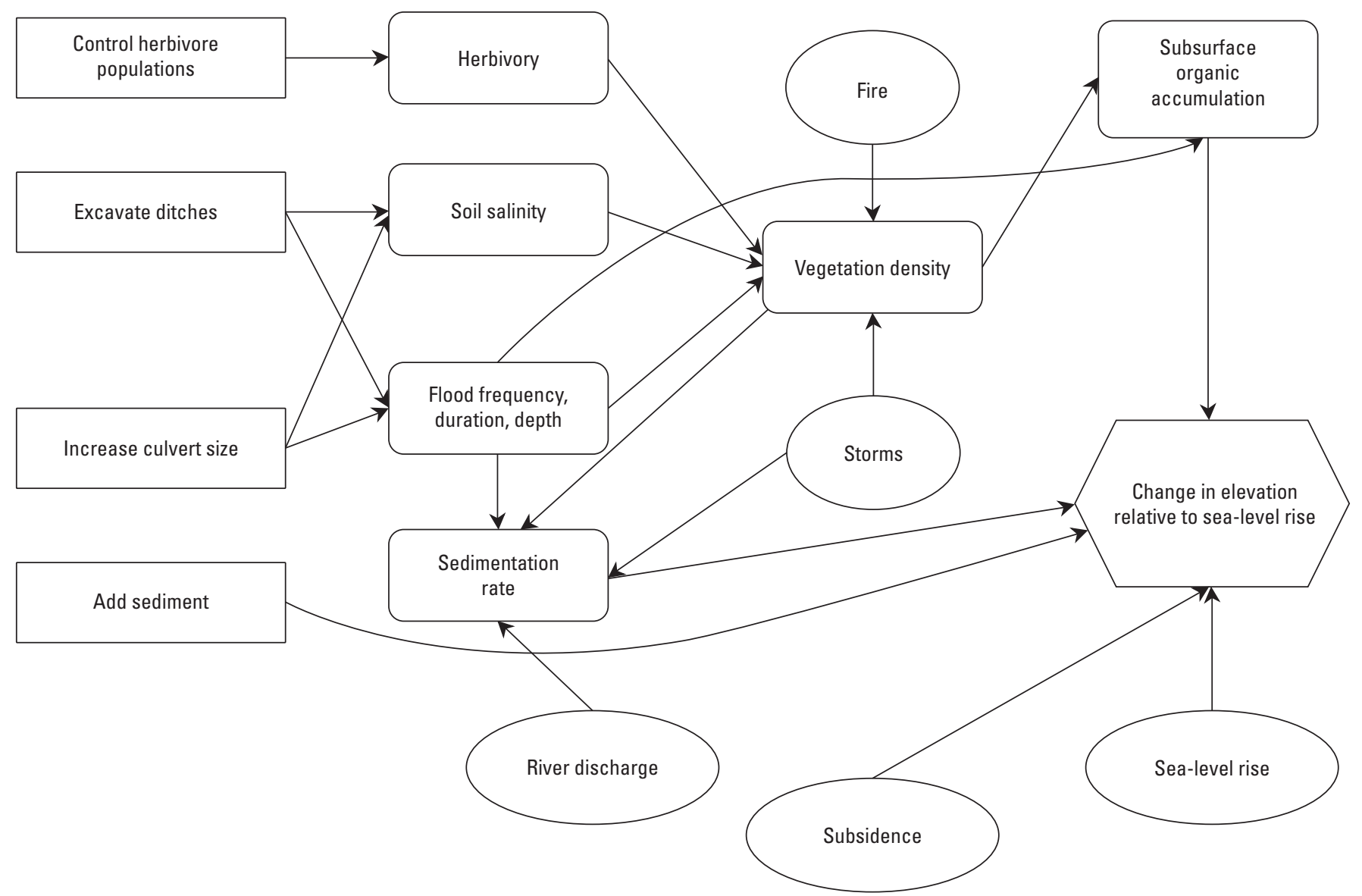

Figure 1.7. Influence diagram used to estimate change in elevation of the marsh surface relative to sea-level rise in response to implementing certain management actions.

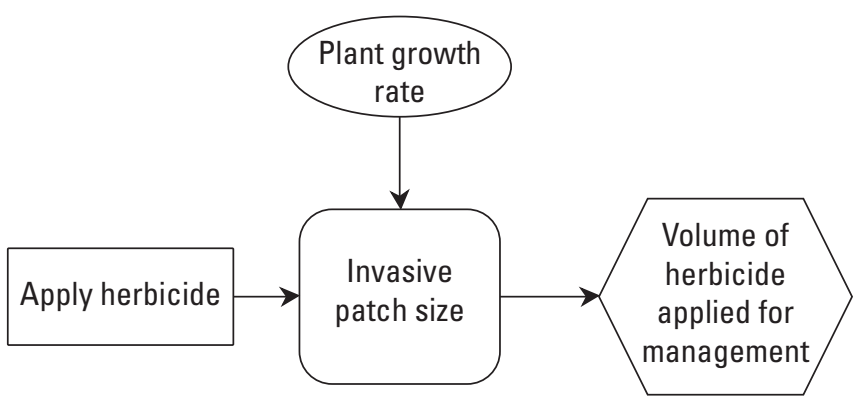

Figure 1.8. Influence diagram used to estimate volume of herbicide that could be applied if a decision was made to use chemical control for removing unwanted vegetation.

\section{Reference Cited}

Neckles, H.A., Lyons, J.E., Guntenspergen, G.R., Shriver, W.G., and Adamowicz, S.C., 2015, Use of structured decision making to identify monitoring variables and management priorities for salt marsh ecosystems: Estuaries and Coasts, v. 38, no. 4, p. 1215-1232. [Also available at https://doi.org/10.1007/s12237-014-9822-5.] 


\section{Appendix 2. Utility Functions for the Eastern Shore of Virginia and Fisherman Island National Wildlife Refuges}

Utilities $[u(x)]$ are derived as monotonically increasing, monotonically decreasing, or step functions over the range of performance metric $x$. In the functions in figures 2.1-2.10, $x$, Low, High, and $\rho$ are expressed in performance metric units; Low and High represent the endpoints of the given metric range for the Eastern Shore of Virginia and Fisherman Island National Wildlife Refuges; and $\rho$ represents a shape parameter derived by stakeholder elicitation (Neckles and others, 2015). Break points in step functions were also derived by stakeholder elicitation.

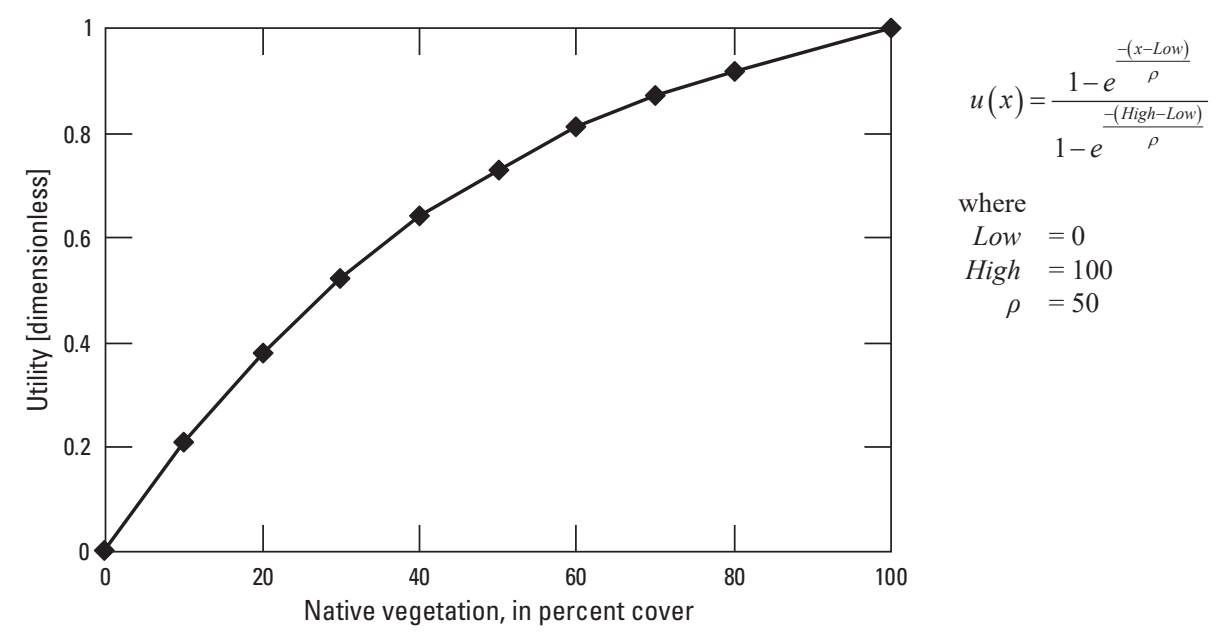

Figure 2.1. Native vegetation at the Eastern Shore of Virginia and Fisherman Island National Wildlife Refuges, Virginia.

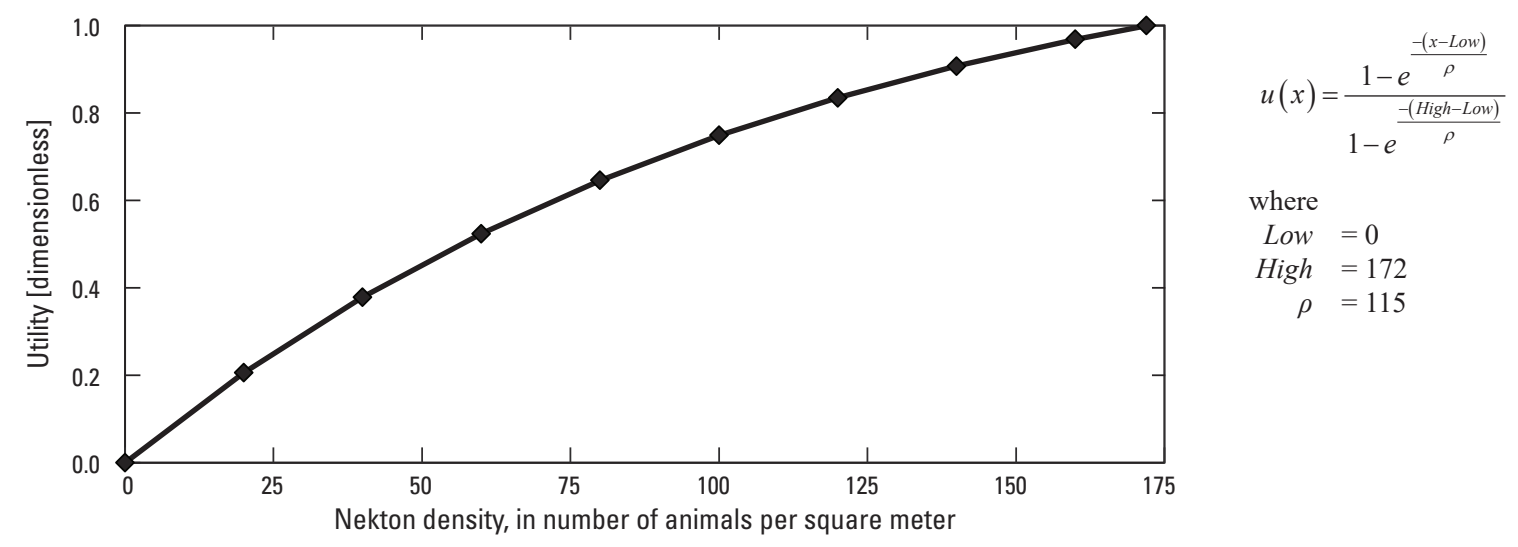

Figure 2.2. Native nekton density at the Eastern Shore of Virginia and Fisherman Island National Wildlife Refuges, Virginia. 


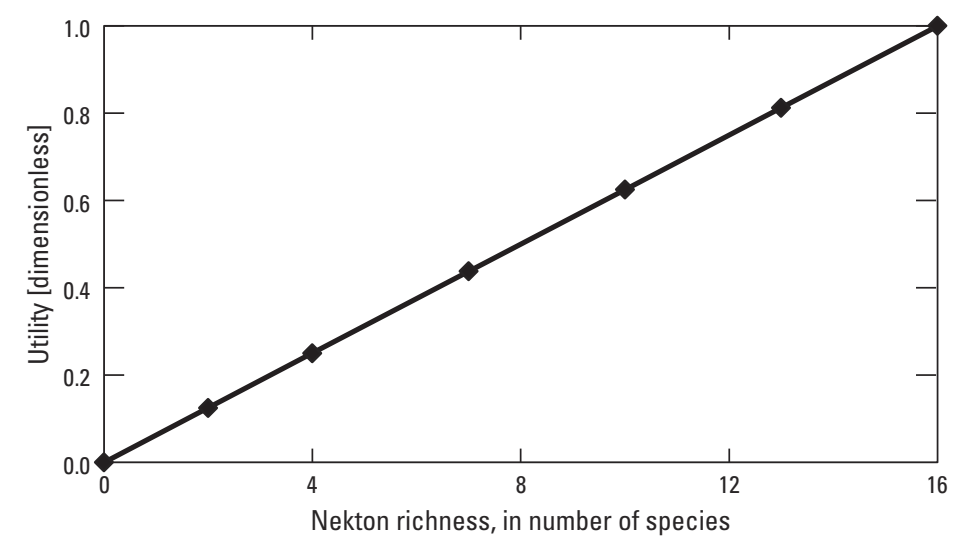

$$
\begin{aligned}
& u(x)=\frac{x-\text { Low }}{\text { High }- \text { Low }} \\
& \text { where } \\
& \begin{aligned}
\text { Low } & =0 \\
\text { High } & =16
\end{aligned}
\end{aligned}
$$

Figure 2.3. Native nekton species richness at the Eastern Shore of Virginia and Fisherman Island National Wildlife Refuges, Virginia.

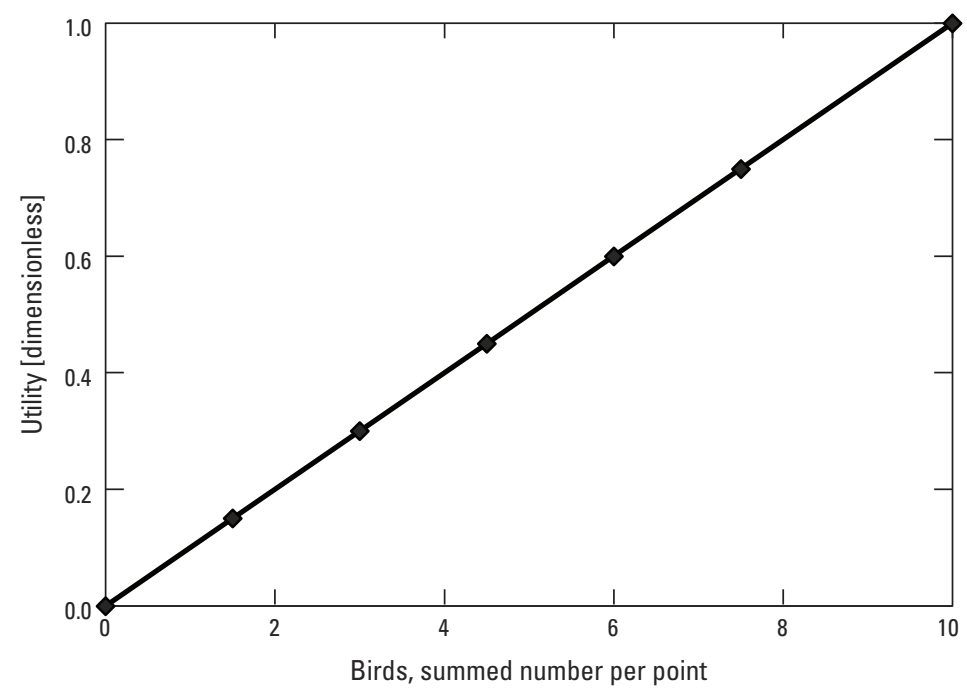

$$
\begin{aligned}
& u(x)=\frac{x-\text { Low }}{\text { High }- \text { Low }} \\
& \text { where } \\
& \begin{aligned}
\text { Low } & =0 \\
\text { High } & =10
\end{aligned}
\end{aligned}
$$

Figure 2.4. Tidal marsh obligate birds at the Eastern Shore of Virginia and Fisherman Island National Wildlife Refuges, Virginia.

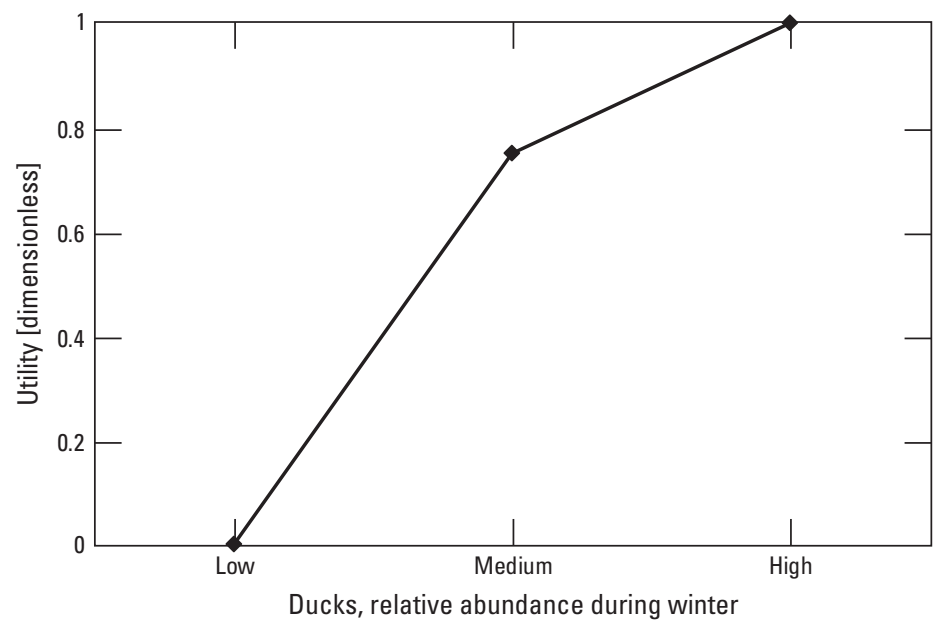

If $x=$ Low, then $u(x)=0$

If $x=$ Medium, then $u(x)$

If $x=$ High, then $u(x)=1$

Figure 2.5. American black ducks at the Eastern Shore of Virginia and Fisherman Island National Wildlife Refuges, Virginia. 


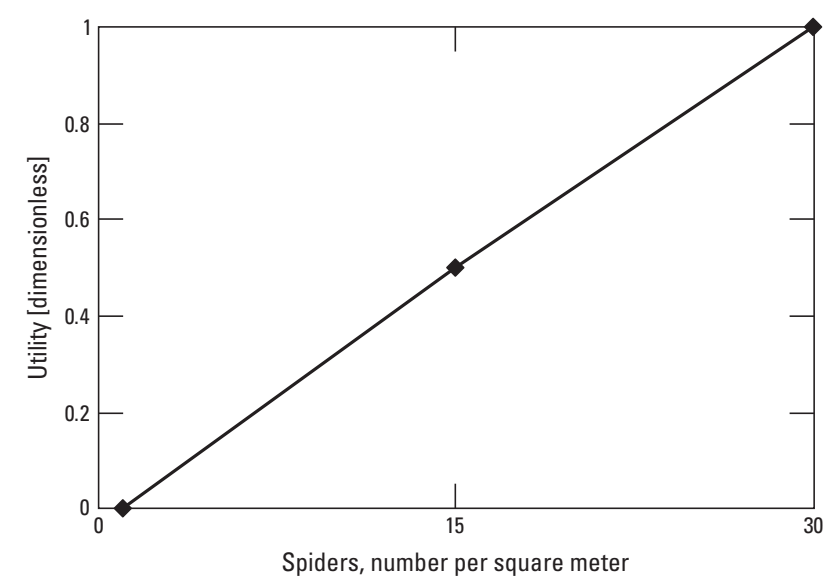

If $x \leq 15$, then $u(x)=0.5 \times \frac{x-1}{14}$
If $x>15$, then $u(x)=0.5+\left(0.5 \times \frac{x-15}{15}\right)$

Figure 2.6. Marsh spiders at the Eastern Shore of Virginia and Fisherman Island National Wildlife Refuges, Virginia.

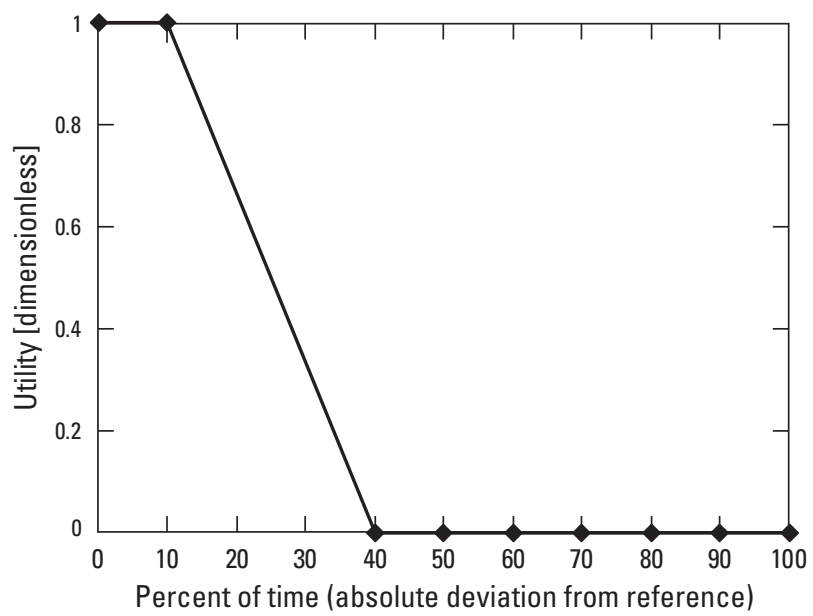

If $x \leq 10$, then $u(x)=1$

If $x \geq 40$, then $u(x)=0$

If $10<x<40$, then $u(x)=1-\frac{x-10}{30}$

Figure 2.7. Duration of surface flooding at the Eastern Shore of Virginia and Fisherman Island National Wildlife Refuges, Virginia.

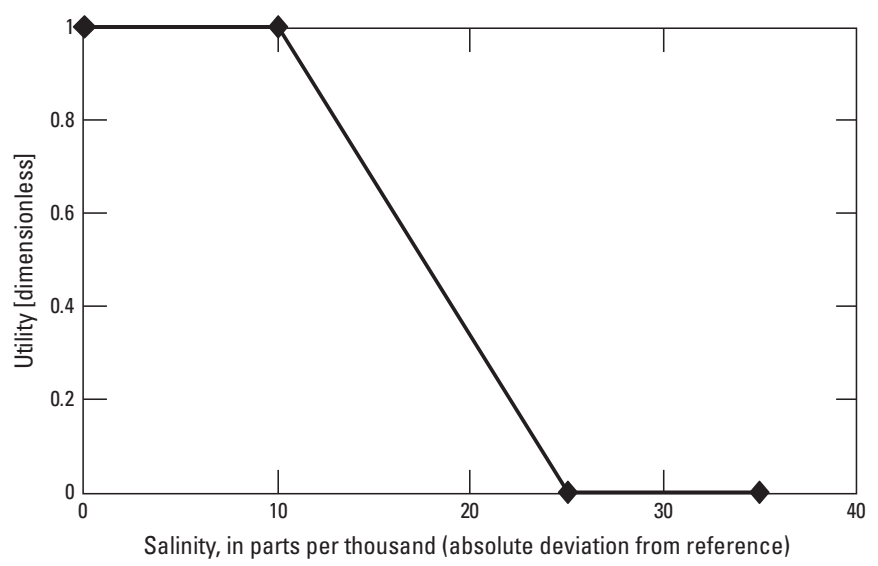

If $x \leq 10$, then $u(x)=1$

If $x \geq 25$, then $u(x)=0$

If $10<x<25$, then $u(x)=1-\frac{x-10}{15}$

Figure 2.8. Salinity of surface water at the Eastern Shore of Virginia and Fisherman Island National Wildlife Refuges, Virginia. 


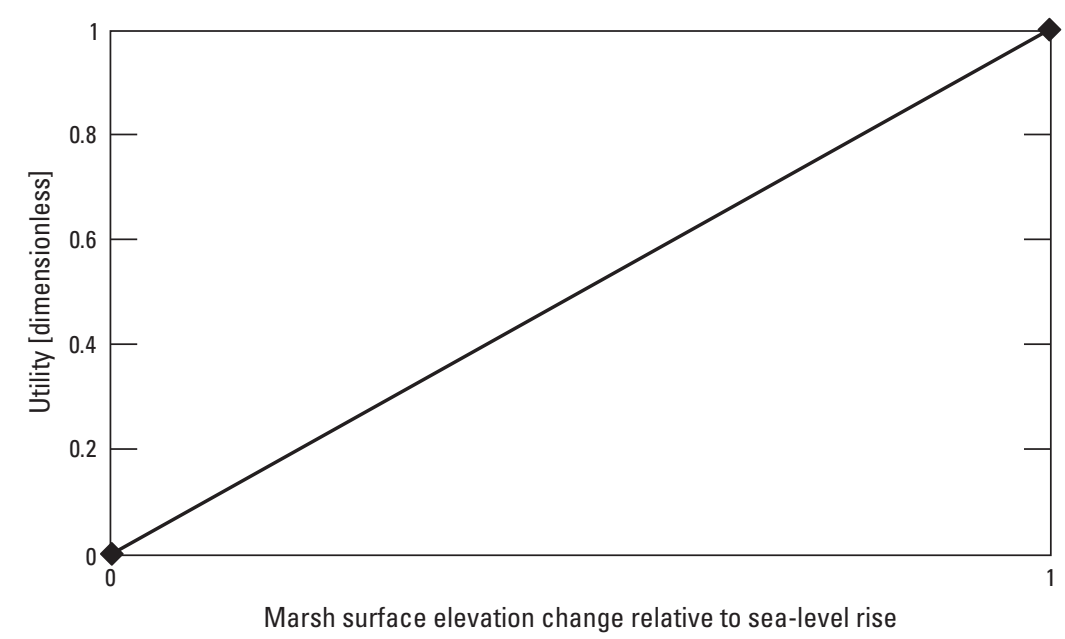

$$
\begin{aligned}
& u(x)=\frac{x-\text { Low }}{\text { High }- \text { Low }} \\
& \text { where } \\
& \qquad \begin{array}{c}
\text { Low } \quad=0 \text {, lower than sea-level rise } \\
\text { High } \quad=1 \text {, above sea-level rise }
\end{array}
\end{aligned}
$$

Figure 2.9. Change in marsh surface elevation relative to sea-level rise at the Eastern Shore of Virginia and Fisherman Island National Wildlife Refuges, Virginia.

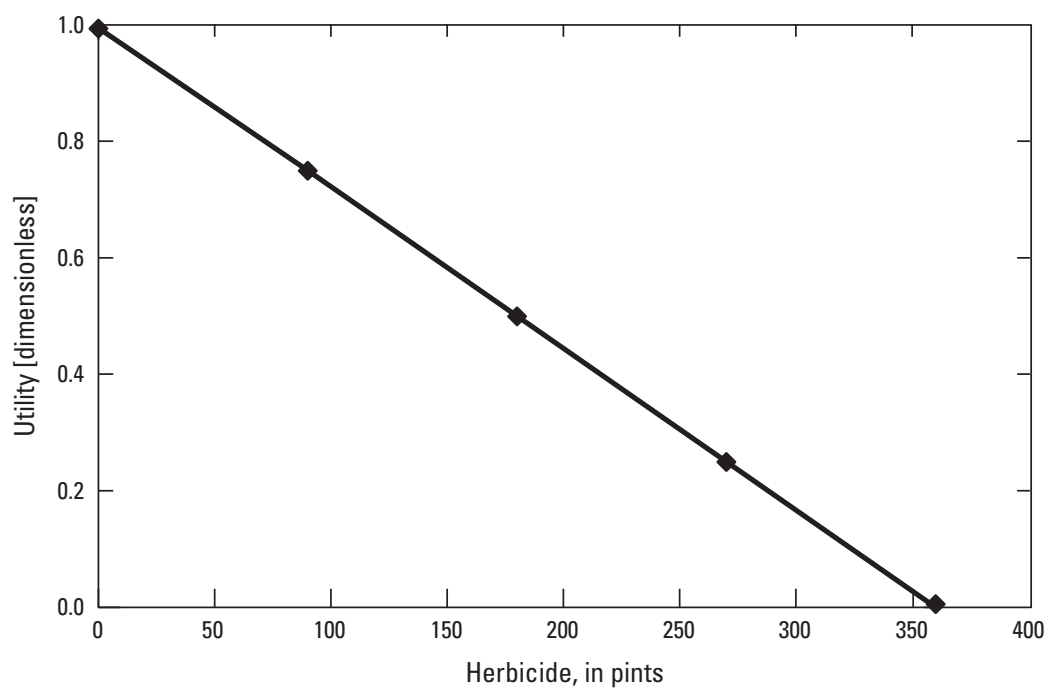

$$
\begin{aligned}
& u(x)=\frac{\text { High }-x}{\text { High }- \text { Low }} \\
& \text { where } \\
& \text { Low } \quad=0 \\
& \text { High }=360
\end{aligned}
$$

Figure 2.10. Application of herbicides at the Eastern Shore of Virginia and Fisherman Island National Wildlife Refuges, Virginia.

\section{Reference Cited}

Neckles, H.A., Lyons, J.E., Guntenspergen, G.R., Shriver, W.G., and Adamowicz, S.C., 2015, Use of structured decision making to identify monitoring variables and management priorities for salt marsh ecosystems: Estuaries and Coasts, v. 38, no. 4, p. 1215-1232. [Also available at https://doi.org/10.1007/s12237-014-9822-5.] 
For more information, contact:

Director, Eastern Ecological Science Center

U.S. Geological Survey

11649 Leetown Road

Kearneysville, WV 25430

https://www.usgs.gov/centers/eesc

Publishing support provided by the

Pembroke Publishing Service Center 
